\title{
STRATIFYING SYSTEMS OVER HEREDITARY ALGEBRAS
}

\author{
PAULA A. CADAVID AND EDUARDO DO N. MARCOS
}

\begin{abstract}
This paper deals with stratifying systems over hereditary algebras. In the case of tame hereditary algebras we obtain a bound for the size of the stratifying systems composed only by regular modules and we conclude that stratifying systems can not be complete. For wild hereditary algebras with more than 2 vertices we show that there exists a complete stratifying system whose elements are regular modules. In the other case, we conclude that there are no stratifing system over them with regular modules. In one example we built all the stratifying systems, with a specific form, having maximal number of regular summads.
\end{abstract}

\section{INTRODUCTION}

Let $A$ be a finite dimensional $K$-algebra. Let $S_{1}, S_{2}, \ldots, S_{n}$ be a complete list of all non-isomorphic simple $A$-modules and we fix this ordering of simple modules. Let $P_{i}$ be the projective cover of $S_{i}$. With this order of simple modules we define for each $i$ the standard module $\triangle_{i}$ to be the maximal quotient of $P_{i}$ with composition factors $S_{j}$ with $j \leq i$. The concept of stratifying system (s.s.) was introduced as a generalization of the standard modules in (Erdmann and Sáenz, 2003). Later, E. Marcos, O. Mendoza and C. Sáenz introduced in (Marcos et al., 2004) and (Marcos et al., 2005) the notion of s.s. via relative simple modules and via relative projective modules, respectively. In these works, the authors show that these concepts are equivalent.

On the other hand, the concept of excepcional sequence was introduced in (Gordentsev and Rudakov, 1987 ) with the aim of to study vector bundles on $\mathbb{P}^{2}$. Such sequences have been also used by several authors in the study of derived categories of algebraic varieties (see for instance (Bondal and Kapranov, 1989), (Rudakov, 1990)). The corresponding notion in the context of representations of a quiver is considered in (Crawley-Boevey, 1993). We observe that over a hereditary algebra, the concept of s.s. and exceptional sequence are equivalent.

The goal of this paper is to study s.s. over hereditary algebras. We introduce the notion of complete stratifying system (c.s.s.) which is a s.s. of maximal possible size and we discuss the existence of a c.s.s. consisting only of regular modules for a hereditary algebra. Every s.s can be extended to a complete one. We calculate all the c.s.s. over the Kronecker algebra and generalized Kronecker algebras. In addition, we construct a family of c.s.s. with a maximal number of regular elements over the canonical algebra $\triangle\left(\widetilde{\mathbb{A}}_{p, q}\right)$.

The paper is organized as follows: We start Section 2 with the main definitions and notations. Section 3 is devoted to the study of the relationship between stratifying systems and tilting modules, using this, we introduce the notion of complete stratifying system. In Section 4 we show give a bound on the size s.s. consisting of regular modules, over a concealed algebra of Euclidean type. In section 5 we show that for any wild hereditary algebra with more than 2 vertices there exists a complete stratifying system whose elements are regular modules. So we can conclude that an algebra, with more than two non isomorphic simple modules, has a c.s.s consisting of regular modules if and only if it is wild. We also exibt in this section the construction of all the c.s.s. over the Kronecker algebra and the generalized Kronecker algebras. In the last section, we construct a s.s. of regular modules with maximal size $(F, G)$ over the canonical algebra $\triangle\left(\widetilde{\mathbb{A}}_{p, q}\right)$, where $F, G$ are sets of simple regular modules. We also extend our construction to a c.s.s. of the form $(X, F, G, Y)$.

Key words and phrases. Stratifying System, Hereditary Algebra, Tilting Module, Standard Hereditary Tube. 


\section{Preliminaries}

The algebras to be considered in this paper are basic, connected and finite dimensional over an algebraically closed field $K$. We denote by $\bmod A$ the category whose objects are finitely generated rigth $A$-modules and by $D$ the usual duality $\operatorname{Hom}_{K}\left(\_, K\right): \bmod A \longrightarrow \bmod A^{o p}$. All the subcategories of $\bmod A$ to be considered are full subcategories.

Let $\mathcal{C}$ be a subcategory of $\bmod A$. We say that $\mathcal{C}$ is

(1) closed under extensions if for every short exact sequence of $A$-modules $0 \rightarrow M_{1} \rightarrow M_{2} \rightarrow M_{3} \rightarrow 0$ with $M_{1}, M_{3} \in \mathcal{C}$, also $M_{2} \in \mathcal{C}$

(2) closed under direct summands provided for every $A$-module $M \in \mathcal{C}$, any direct summand of $M$ belongs to $\mathcal{C}$;

(3) closed under kernels of epimorphisms if for every $A$-module epimorphism $f: M \rightarrow N$ with $M, N \in \mathcal{C}$, also $\operatorname{ker}(f) \in \mathcal{C}$.

Given a finite set $X$ of modules, we denote by $\mathcal{F}(X)$ the subcategory of $\bmod A$ containing the zero module and all modules which are filtered by modules in $X$. That is, a non zero module $M$ belongs to $\mathcal{F}(X)$ when there is a finite chain

$$
0=M_{0} \subseteq M_{1} \subseteq \ldots \subseteq M_{t}=M
$$

of submodules of $M$ such that $M_{i} / M_{i-1}$ is isomorphic to a module in $X$, for all $i=1,2, \ldots, t$. Other category related with $\mathcal{F}(X)$ is $\mathcal{Y}(X)$ whose objects are the modules $M \in \bmod A$ such that $\left.\operatorname{Ext}_{A}^{1}(-, M)\right|_{\mathcal{F}(X)}=0$.

We introduce now the main notion in this work.

Definition 2.1. (Erdmann and Sáenz, 2003) Let $X=\left\{X_{i}\right\}_{i=1}^{t}$ be a set of non-zero A-modules and $\underline{Y}=$ $\left\{Y_{i}\right\}_{i=1}^{t}$ be a set of indecomposable A-modules. The pair $(X, \underline{Y})$ is a stratifying system (s.s.) of size $t$, if the following three conditions hold:

(1) $\operatorname{Hom}_{A}\left(X_{j}, X_{i}\right)=0$, for $j>i$.

(2) For each $i \in\{1, \ldots, t\}$ there is an exact sequence $0 \rightarrow X_{i} \rightarrow Y_{i} \rightarrow Z_{i} \rightarrow 0$, where $Z_{i} \in \mathcal{F}\left(X_{1}, \ldots, X_{i-1}\right)$.

(3) $\left.\operatorname{Ext}_{A}^{1}\left(\_, Y\right)\right|_{\mathcal{F}(X)}=0$, where $Y=\coprod_{i=1}^{t} Y_{i}$.

The modules in the set $X$ will be called relative simple modules in $\mathcal{F}(X)$. This nomenclature is justified by the fact that they are exactly the modules which do have not proper submodules in the category $\mathcal{F}(X)$. In order to give a characterization of stratifying systems depending only on the set of relative simple modules we will make use of the following result.

Proposition 2.2. (Erdmann and Sáenz, 2003) Given a set $X=\left\{X_{i}\right\}_{i=1}^{t}$ of non-zero A-modules the following conditions are equivalent:

(1) There exists a set of indecomposable A-modules $\underline{Y}=\left\{Y_{i}\right\}_{i=1}^{t}$ such that $(X, \underline{Y})$ is a stratifying system of size $t$.

(2) The set $X$ satisfies the following conditions:

(a) $\operatorname{Hom}_{A}\left(X_{j}, X_{i}\right)=0$, for $j>i$.

(b) $\operatorname{Ext}_{A}^{1}\left(X_{j}, X_{i}\right)=0$, for $j \geq i$.

(c) $X_{i}$ is indecomposable, for all $i=1,2, \ldots, t$.

As a consequence of the previous result we have the following alternative definition of stratifying systems.

Definition 2.3. (Marcos et al., 2004) A stratifying system (s.s.) of size $t$ consist of a set $X=\left\{X_{i}\right\}_{i=1}^{t}$ of indecomposable $A$-modules satisfying the following conditions:

(1) $\operatorname{Hom}_{A}\left(X_{j}, X_{i}\right)=0$, for $j>i$.

(2) $\operatorname{Ext}_{A}^{1}\left(X_{j}, X_{i}\right)=0$, for $j \geq i$.

Given a s.s. $X$ and an $A$-module $M \in \mathcal{F}(X)$, we denote by $\left[M: X_{i}\right]$ the multiplicity of the $A$-module $X_{i}$ on a given filtration of $M$. The number $\left[M: X_{i}\right]$ is well defined, that is, it does not depend on a given filtration of $M$ in $X$ (see (Erdmann and Sáenz, 2003)). The category $\mathcal{F}(X)$ is closed by direct summads, under extensions and funtorially finite $\operatorname{in} \bmod A$, so it has (relative) almost split sequences (see (Ringel, 1991)). On the other hand, it is not necessarily closed under kernels of epimorphisms (see (Marcos et al., 2004)). 
Let $A$ be a hereditary $K$-algebra. An $A$-module $X$ is called exceptional if $\operatorname{End}_{A}(X) \cong K$ and $\operatorname{Ext}_{A}^{1}(X, X)=$ 0 and a sequence $X=\left(X_{1}, \ldots, X_{t}\right)$ is called exceptional if it is a sequence of exceptional modules satisfaying $\operatorname{Hom}_{A}\left(X_{i}, X_{j}\right)=0$, for $i>j$ and $\operatorname{Ext}_{A}^{1}\left(X_{i}, X_{j}\right)=0$ for $i \geq j$. A sequence $X=\left(X_{1}, \ldots, X_{n}\right)$ is said to be complete if $n$ is the number of isomorphism classes of simple $A$-modules.

We observe that if $A$ is a hereditary $K$-algebra, then the sequence of $A$-modules $\left(X_{1}, \ldots, X_{t}\right)$ is an exceptional sequence if, and only if, the set $X=\left\{X_{i}\right\}_{i=1}^{t}$ is a s.s. over $A$.

\section{STRATIFYING SYSTEMS OVER HEREDitARY ALGEBRAS AND TILTING MODULES}

We recall that an $A$-module $T$ is called partial tilting if the following conditions hold:

T1. $\operatorname{pd} T \leq 1$,

T2. $\operatorname{Ext}_{A}^{1}(T, T)=0$.

A partial tilting module $T$ is called tilting module if it satisfies the following additional condition:

T3. There exist a short exact sequence $0 \rightarrow A_{A} \rightarrow T_{1} \rightarrow T_{2} \rightarrow 0$, with $T_{1}, T_{2}$ in add $T$, where add $T$ is the subcategory of $\bmod A$ whose objects are direct sums of direct summands of $T$.

Finally, a tilting $A$-module $T$ is called basic tilting module if the number of pairwise non-isomorphic indecomposable summands of $T$ is the rank of $K_{0}(A)$.

Lemma 3.1. Let $Q$ be a finite, connected and acyclic quiver with $n$ vertices, $A=K Q$ and $X=\left\{X_{i}\right\}_{i=1}^{t}$ be a s.s. over $A$. Then $\mathcal{F}(X) \cap \mathcal{Y}(X)=\operatorname{add} T$, where $T$ is a basic partial tilting $A$-module, and $t \leq n$.

Proof. According to Theorem 2.4 in (Marcos et al., 2004) we have that $\mathcal{F}(X) \cap \mathcal{Y}(X)=\operatorname{add} Y$, where $Y$ is an $A$-module with $t$ pairwise non-isomorphic indecomposable direct summands and such that $\operatorname{Ext}_{A}^{1}(Y, Y)=0$. This means that $Y$ is a partial tilting module and therefore we conclude that $t \leq n$.

In the previous lemma the hypothesis that $A$ is a hereditary algebra is essential as the following example shows.

Example 3.2. (Marcos et al., 2004) Let $A$ be the path algebra of the quiver $3 \stackrel{\alpha}{\longrightarrow} 1 \stackrel{\beta}{\longleftarrow} 2 \stackrel{\gamma}{\longleftarrow} 4$ bound by $\gamma \beta$. Taking $X_{1}=S_{1}=P_{1}, X_{2}=P_{2}, X_{3}=P_{3}, X_{4}=P_{4}=I_{2}$ and $X_{5}=S_{4}$ we have that $X=$ $\left\{X_{1}, X_{2}, X_{3}, X_{4}, X_{5}\right\}$ is a s.s. of size 5 .

This motivates the following definition.

Definition 3.3. Let $Q$ be a finite, connected and acyclic quiver with $n$ vertices and $A=K Q$. A s.s. $X=\left\{X_{i}\right\}_{i=1}^{t}$ over $A$ is called complete (c.s.s.) if $t=n$.

Let $Q=\left(Q_{0}, Q_{1}\right)$ be a quiver such that $\left|Q_{0}\right|=n$. An admissible numbering of $Q$ is a bijection between $Q_{0}$ and the set $\{1,2, \ldots n\}$ such that, if we have an arrow $j \rightarrow i$ then $j>i$. Let us note that a connected quiver $Q$, with more than one vertice, is an acyclic quiver if and only if there exist an admissible numbering of $Q$.

Proposition 3.4. Let $A$ be a hereditary $K$-algebra and $T$ be a basic tilting $A$-module. Then there exists a numbering of the sumands of $T=\coprod_{i=1}^{n} T_{i}$ such that $\left(T_{1}, \ldots, T_{n}\right)$ is a c.s.s.

Proof. Let $B=\operatorname{End}_{A}(T)$. Then the ordinary quiver $Q_{B}$ of $B$ is acyclic. If $\boldsymbol{e}=\left(e_{1}, \ldots, e_{n}\right)$ is an admissible ordering of the vertices of $Q_{B}$ and we take $P_{i}=e_{i} B$ then $\operatorname{Hom}_{B}\left(P_{i}, P_{j}\right)=0$, for $j<i$. So $\left(P_{1}, \ldots, P_{n}\right)$ is a s.s. over $B$. Since $\operatorname{Hom}_{A}(T,-):$ add $T \longrightarrow \mathcal{P}$, when $\mathcal{P}$ is the category of projective $B$-modules, is an equivalence of categories then $\operatorname{Hom}_{A}\left(T_{i}, T_{j}\right)=0$, for $j<i$. But $\operatorname{Ext}_{A}^{1}\left(T_{i}, T_{j}\right)=0$, for all $i, j$, then we get that $\left(T_{1}, \ldots, T_{n}\right)$ is a c.s.s.

\section{S.S OVER HEREDITARY STANDARD STABLE TUBES}

We start this section by collecting some preliminary facts about hereditary standard stable tubes needed latter in this section. The facts which we describe next can be found in (Simsom and Skowronski, 2007, Vol. 2 and Vol. 3) and we include here for the sake of completeness.

We recalling that a standard tube $\mathcal{T}$, of the Auslander-Reiten quiver of an algebra $A$, is hereditary when $\operatorname{pd} X \leq 1$ and id $X \leq 1$, for any module $X$ of $\mathcal{T}$. 
Let $A$ be an algebra, $\mathcal{T}_{\lambda}$ be a fixed hereditary standard stable tube of $\operatorname{rank} r_{\lambda}$ in $\Gamma(\bmod A)$. The set of modules having exactly one immediate predecessor in $\mathcal{T}_{\lambda}$ is called the mouth of $\mathcal{T}_{\lambda}$. Given a module $X$ lying on the mouth of $\mathcal{T}_{\lambda}$, a ray starting at $X$ is defined to be the unique infinite sectional path $X=$ $X[1] \rightarrow X[2] \rightarrow X[3] \rightarrow \cdots \rightarrow X[m] \rightarrow \cdots$ in $\mathcal{T}_{\lambda}$.

Let $E_{1}, E_{2} \ldots, E_{r_{\lambda}}$ be the $A$-modules lying on the mouth of $\mathcal{T}_{\lambda}$ which form a $\tau_{A}$-cycle $\left(E_{1}, E_{2} \ldots, E_{r_{\lambda}}\right)$, that is, they are ordered in such a way that $\tau E_{2} \cong E_{1}, \tau E_{3} \cong E_{2}, \ldots, \tau E_{r_{\lambda}} \cong E_{r_{\lambda}-1}, \tau E_{1} \cong E_{r_{\lambda}}$. Then

- the modules $E_{1}, E_{2} \ldots, E_{r_{\lambda}}$ are pairwise orthogonal bricks in $\bmod A$;

- every indecomposble $A$-module $M$ in $\mathcal{T}_{\lambda}$ is of the form $M \cong E_{j}[m]$, for some $j \in\left\{1, \ldots, r_{\lambda}\right\}$ and $m \geq 1$

- the subcategory add $\mathcal{T}_{\lambda}$ of $\bmod A$ is hereditary, abelian and the modules $E_{1}, E_{2} \ldots, E_{r_{\lambda}}$ form a complete set of pairwise non-isomorphic simple objects of add $\mathcal{T}_{\lambda}$;

- the module $E_{i}=E_{i}[1]$ is the unique simple subobject of $M \cong E_{i}[j]$, and $j$ is the length $\ell_{\lambda}(M)$ of $X$ in $\operatorname{add} \mathcal{T}_{\lambda}$.

For any indecomposable $A$-module $M \cong E_{i}[j]$ we denote by $\mathcal{C}(M)$ the cone in the tube $\mathcal{T}_{\lambda}$ determined by $M$. That is, $\mathcal{C}(M)$ is the full translation subquiver of $\mathcal{T}_{\lambda}$ of the form

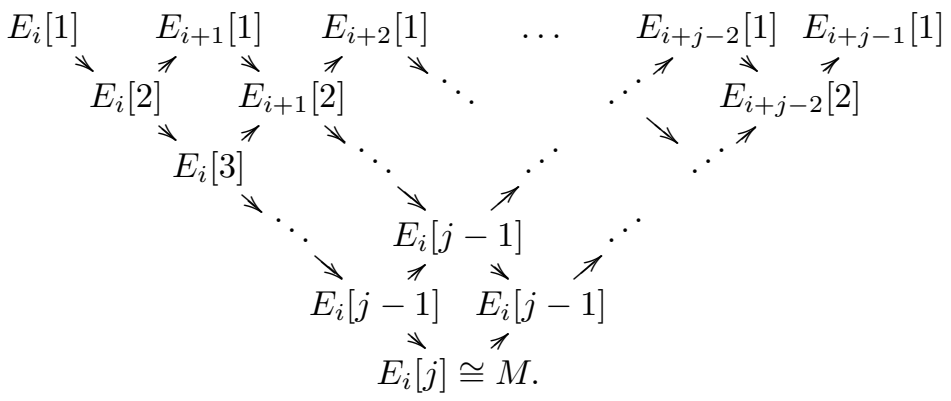

Lemma 4.1. Let $A$ be an algebra and $\mathcal{T}_{\lambda}$ a hereditary standard stable tube of $\Gamma(\bmod A)$ of rank $r_{\lambda} \geq 1$. If $N_{1}, \ldots, N_{t}$ are parwise non-isomorphic indecomposable $A$-modules such that $\operatorname{Ext}_{A}^{1}\left(N_{1} \oplus \ldots \oplus N_{t}, N_{1} \oplus \ldots \oplus\right.$ $\left.N_{t}\right)=0$ and $\mathcal{C}\left(N_{i}\right) \cap \mathcal{C}\left(N_{j}\right)=\emptyset$, for $i \neq j$, then $\ell_{\lambda}\left(N_{1}\right)+\cdots+\ell_{\lambda}\left(N_{t}\right) \leq r_{\lambda}-t$.

Proof. Let $\left(X_{1}, \ldots, X_{r_{\lambda}}\right)$ be a $\tau$-cycle of mouth modules of the tube $\mathcal{T}_{\lambda}$. We denote by $\mathcal{N}_{i}$ and $\mathcal{N}_{i}^{*}$ the sets $\mathcal{N}_{i}=\mathcal{C}\left(\mathcal{N}_{i}\right) \cap\left\{X_{1}, \ldots X_{r_{\lambda}}\right\}$ and $\mathcal{N}_{i}^{*}=\tau \mathcal{C}\left(N_{i}\right) \cap\left\{X_{1}, \ldots, X_{r_{\lambda}}\right\}$. If $N_{i} \cong X_{l_{i}}\left[t_{i}\right]$, then

$$
\mathcal{N}_{i}=\left\{X_{l_{i}}[1], \ldots, X_{l_{i}+t_{i}-1}[1]\right\} \text { and } \mathcal{N}_{i}^{*}=\left\{X_{l_{i}-1}[1], \ldots, X_{l_{i}+t_{i}-2}[1]\right\} \text {. }
$$

Therefore $\left|\mathcal{N}_{i} \cup \mathcal{N}_{i}^{*}\right|=t_{i}+1=\ell_{\lambda}\left(N_{i}\right)+1$. It follows from the hypothesis and from Lemma 1.7 in (Simsom and Skowronski, 2007, Vol. 3, Chap. XVII) that $\mathcal{C}\left(N_{i}\right) \cap \tau \mathcal{C}\left(N_{j}\right)=\emptyset$ and $\tau \mathcal{C}\left(N_{i}\right) \cap \mathcal{C}\left(N_{j}\right)=\emptyset$. Hence

$$
\left|\bigcup_{i=1}^{t}\left(\mathcal{N}_{i} \cup \mathcal{N}_{i}^{*}\right)\right|=\left(\sum_{i=1}^{t} \ell_{\lambda}\left(N_{i}\right)\right)+t .
$$

On the other hand, we have $\bigcup_{i=1}^{t}\left(\mathcal{N}_{i} \cup \mathcal{N}_{i}^{*}\right) \subseteq\left\{X_{1}, \ldots, X_{r_{\lambda}}\right\}$. Then $\sum_{i=1}^{t} \ell_{\lambda}\left(N_{i}\right) \leq r_{\lambda}-t$.

Proposition 4.2. Let $A$ be an algebra and $\mathcal{T}_{\lambda}$ a hereditary standard stable tube of $\Gamma(\bmod A)$ of rank $r_{\lambda} \geq 1$. If $T$ is a multiplicity-free partial tilting $A$-module, then $T$ has at most $r_{\lambda}-1$ direct summands in add $\mathcal{T}_{\lambda}$.

Proof. Let $T$ be a partial tilting $A$-module. We define sets $\mathcal{X}$ and $\mathcal{X}_{0}$ as follow

$$
\begin{aligned}
\mathcal{X} & =\left\{X: X \in \operatorname{add} \mathcal{T}_{\lambda} \cap \text { add } T \cap \text { ind } A\right\} \text { and } \\
\mathcal{X}_{0} & =\{X \in \mathcal{X}: \forall Y \in \mathcal{X}, \mathcal{C}(Y) \subseteq \mathcal{C}(X) \text { or } \mathcal{C}(X) \cap \mathcal{C}(Y)=\emptyset\}
\end{aligned}
$$

Since $T$ is a multiplicity-free partial tilting $A$-module hence $\mathcal{X}$ and $\mathcal{X}_{0}$ are finite. We note that if $X_{1}, X_{2} \in \mathcal{X}_{0}$ and $X_{1} \neq X_{2}$, then $\mathcal{C}\left(X_{1}\right) \cap \mathcal{C}\left(X_{2}\right)=\emptyset$. Therefore if $\left|\mathcal{X}_{0}\right|=\ell$, by 4.1, we have $\sum_{X \in \mathcal{X}_{0}} \ell_{\lambda}(X) \leq r_{\lambda}-\ell$. Furthermore, if $X \in \mathcal{X}_{0}$ then, by 1.6 (Simsom and Skowronski, 2007, Vol. 3, Chap. XVII), $|\mathcal{C}(X) \cap \mathcal{X}| \leq$ $\ell_{\lambda}(X)$. Thus

$$
|\mathcal{X}| \leq \sum_{X \in \mathcal{X}_{0}}|\mathcal{C}(X) \cap \mathcal{X}| \leq \sum_{X \in \mathcal{X}_{0}} \ell_{\lambda}(X) \leq r_{\lambda}-\ell
$$

and we conclude that if $\mathcal{X} \neq \emptyset$ then $|\mathcal{X}| \leq r_{\lambda}-1$. 
Proposition 4.3. Let $A$ be an algebra and $\mathcal{T}_{\lambda}$ a hereditary standard stable tube of $\Gamma(\bmod A)$ of rank $r_{\lambda} \geq 1$. Then there exist a s.s. of size $r_{\lambda}-1$ lying in $\mathcal{T}_{\lambda}$. Further, if $X=\left\{X_{1}, \ldots, X_{t}\right\}$ is a s.s. such that $X_{i} \in \operatorname{add} \mathcal{T}_{\lambda}$, for all $i=1, \ldots, t$, then $t \leq r_{\lambda}-1$.

Proof. Let $\left(X_{1}, \ldots, X_{r_{\lambda}}\right)$ be a $\tau$-cycle of modules lying on the mouth of $\mathcal{T}_{\lambda}$. Using the Auslander formula, we get that if $1<i \leq j \leq r_{\lambda}-1$ then $\operatorname{Ext}_{A}^{1}\left(X_{i}, X_{j}\right) \cong D \operatorname{Hom}_{A}\left(X_{j}, \tau X_{i}\right) \cong D \operatorname{Hom}_{A}\left(X_{j}, X_{i-1}\right)$. Since $j \neq i-1$ we see that $\operatorname{Hom}_{A}\left(X_{j}, X_{i-1}\right)=0$. Therefore, $\operatorname{Ext}_{A}^{1}\left(X_{i}, X_{j}\right)=0$. If $i=1$ and $1 \leq j \leq r-1$, again by the Auslander formula, we have $\operatorname{Ext}_{A}^{1}\left(X_{1}, X_{j}\right) \cong D \operatorname{Hom}_{A}\left(X_{j}, \tau X_{1}\right) \cong D \operatorname{Hom}_{A}\left(X_{j}, X_{r_{\lambda}}\right)$. Because $j \neq r$ it follows that $\operatorname{Hom}_{A}\left(X_{j}, X_{r}\right)=0$. Thus $\operatorname{Ext}_{A}^{1}\left(X_{1}, X_{j}\right)=0$. This implies that $X=\left(X_{r_{\lambda}-1}, \ldots, X_{1}\right)$ is a s.s. of size $r_{\lambda}-1$.

Now suppose that $Y=\left\{Y_{1}, \ldots, Y_{t}\right\}$ is a s.s. such that $Y_{i} \in$ add $\mathcal{T}_{\lambda}$. As add $\mathcal{T}_{\lambda}$ is abelian and extension closed subcategory of $\bmod A$ then $\mathcal{F}(Y) \subseteq$ add $\mathcal{T}_{\lambda}$. On the other hand, by 3.1, $\mathcal{F}(Y) \cap \mathcal{Y}(Y)=$ add $T$, where $T$ are a partial tilting $A$-module and then, by 4.2, the number of indecomposable direct summands of $T$ are at almost $r_{\lambda}-1$. Because the size of $X$ is equal to a number of indecomposable direct summands of $T$ we have that $t \leq r_{\lambda}-1$.

Let $Q$ be a finite, connected and acyclic quiver that is not a Dynkin quiver. An algebra $B$ is called concealed of type $\mathbf{Q}$ if there exists a postprojective tilting module $T$ over the path algebra $A=K Q$ such that $B=\operatorname{End}_{A}\left(T_{A}\right)$.

Note that if $A=K Q$ and $Q$ is an Euclidean quiver then $A$ is a concealed algebra of type $Q$. We recall from (Simsom and Skowronski, 2007, Vol. 2) that, if $B=\operatorname{End}_{A}\left(T_{A}\right)$ is a concealed algebra of Euclidean type then $\operatorname{pd} Z \leq 1$ and id $Z \leq 1$, for all but finitely many non-isomorphic indecomposable $B$-modules $Z$ that are postprojective or preinjective. The category $\mathcal{R}(B)$, whose objects are all the regular $B$-modules, is abelian and closed by extensions and the components of $\mathcal{R}(B)$ are a family $\mathcal{T}^{B}=\left\{\mathcal{T}_{\lambda}^{B}\right\}_{\lambda \in \Lambda}$ of pairwise orthogonal standard stable and hereditary tubes. Moreover, if $r_{\lambda}^{B} \geq 1$ denotes the rank of $\mathcal{T}_{\lambda}^{B}$ and $n$ is the rank of $K_{0}(A)$, then

$$
\sum_{\lambda \in \Lambda} r_{\lambda}^{B}-1 \leq n-2
$$

Using the properties of concealed algebras mentioned above and 4.3 we have the following result.

Theorem 4.4. Let $A$ be a concealed algebra of Euclidean type. If $X$ is a regular s.s. of size $t$ over $A$ then $t \leq \operatorname{rank} K_{0}(A)-2$. In particular, if $A$ is a hereditary algebra of Euclidean type there is no a regular c.s.s. over $A$.

\section{S.S. OVER WILD HEREDITARY ALGEBRAS}

In this section we describe all the c.s.s. over the Kronecker algebra and the generalized Kronecker algebras, and give a caracterization of wild hereditary algebras in terms of stratifying systems.

We begin by stating some known statements, which we will use.

Lemma 5.1. (Crawley-Boevey, 1993) Any exceptional sequence $\left(X_{1}, \ldots, X_{a}, Z_{1}, \ldots, Z_{c}\right)$ can be enlarged to a complete sequence $\left(X_{1}, \ldots, X_{a}, Y_{1}, \ldots, Y_{b}, Z_{1}, \ldots, Z_{c}\right)$.

Lemma 5.2. (Crawley-Boevey, 1993) If $E=\left(X_{1}, \ldots, X_{n}\right)$ and $F=\left(Y_{1}, \ldots, Y_{n}\right)$ are complete exceptional sequences such that $X_{j} \cong Y_{j}$ for $j \neq i$, then $E=F$.

Proposition 5.3. Let $A=K Q$, where $Q$ is the quiver

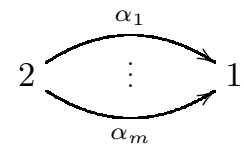

with $m \geq 2$. Then the list of all c.s.s. over $A$ is the following:

(1) $\left(I_{2}, P_{1}\right)$.

(2) $\left(\tau^{-i} P_{1}, \tau^{-i} P_{2}\right)$, with $i \geq 0$.

(3) $\left(\tau^{-i} P_{2}, \tau^{-i-1} P_{1}\right)$, with $i \geq 0$. 
(4) $\left(\tau^{i} I_{1}, \tau^{i} I_{2}\right)$, with $i \geq 0$.

(5) $\left(\tau^{i+1} I_{2}, \tau^{i} I_{1}\right)$, with $i \geq 1$.

Proof. First we observe that independently of $m$ all the indecomposable regular modules over $A$ have selfextensions. In fact, let $M$ be a regular indecomposable $A$-module. If $m=2$ then $M$ lives in an homogeneous tube of $\Gamma(\bmod A)$, so it has self extension, at least the one given by the almost split sequence. If $m>2$, as a consequence of 2.16 in (Simsom and Skowronski, 2007, Vol. 3, Chap. XVII), $M$ has non trivial self-extensions. So all stratifying systems consist only of posprojetive or preinjetive modules.

Just as an example, we will show that the pair (2) in the list is a s.s.. The other verifications are similar. Using the Auslander formula we have the isomorphism $\operatorname{Hom}_{A}\left(\tau^{-i} P_{2}, \tau^{-i} P_{1}\right) \cong \operatorname{Hom}_{A}\left(P_{2}, P_{1}\right)$. But $P_{1}$ is a simple projetive module and then $\operatorname{Hom}_{A}\left(P_{2}, P_{1}\right)=0$. On the other hand, using the Auslander formula again, we have that $\operatorname{Ext}_{A}^{1}\left(\tau^{-i} P_{2}, \tau^{-i} P_{1}\right) \cong D \operatorname{Hom}_{A}\left(\tau^{-i} P_{1}, \tau^{-i+1} P_{2}\right) \cong D \operatorname{Hom}_{A}\left(\tau^{-1} P_{1}, P_{2}\right)=0$. Therefore $\left(\tau^{-i} P_{1}, \tau^{-i} P_{2}\right)$, with $i \geq 0$, is a c.s.s. over $A$.

Finally we must guarantee that the list is complete. Observe that all posprojective and all preinjective appear as the first entry on exactly one of the pairs described by the list, so the result follow by 5.1 .

Corollary 5.4. Let $A$ be as in the previous theorem and $X$ be a c.s.s. over $A$. The category $\mathcal{F}(X)$ is infinite if, and only if, $X=\left(I_{2}, P_{1}\right)$.

Proof. Since $I_{2}=S_{2}$ and $P_{1}=S_{1}$ then $\mathcal{F}\left(I_{2}, P_{1}\right)=\bmod A$. In the other cases, by using Lemma 6.3 can be shown that $\mathcal{F}\left(X_{1}, X_{2}\right)=\operatorname{add}\left(X_{1} \oplus X_{2}\right)$.

We recall that if $Q$ is a finite connected acyclic quiver and $A=K Q$ then there exists a regular tilting module in $\bmod A$ if, and only if, $Q$ is neither a Dynkin or a Euclidean quiver, and $Q$ has at least three vertices (see (Baer, 1989)). Therefore, by 4.4 and 5.3 we have the main result of this section which is stated in the following theorem.

Theorem 5.5. Let $Q$ be a finite connected acyclic quiver, $\left|Q_{0}\right| \geq 3$ and $A=K Q$. There exists a regular c.s.s over $A$ if, and only if, $A$ is a wild hereditary algebra.

\section{Stratifying Systems over algebras of type $\Delta\left(\widetilde{\mathbb{A}}_{p, q}\right)$}

In this section $p$ and $q$ are integers such that $1 \leq p \leq q$. Let $\Delta=\Delta\left(\widetilde{\mathbb{A}}_{p, q}\right)$ be the canonically oriented Euclidean quiver

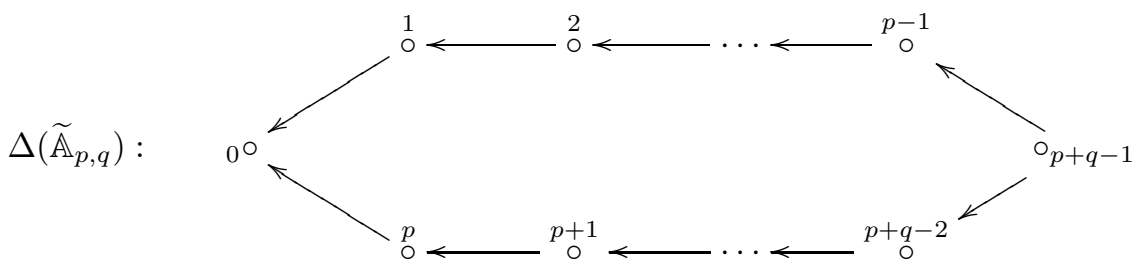

We give a construction of s.s over $K \Delta$ with a maximal number of regular modules. To start we need to recall the description of the simple regular representations of $K \Delta$, and their behavior under $\tau$, which we give next.

(a) Simple regular representations in the tube $\mathcal{T}_{\infty}^{\Delta}$

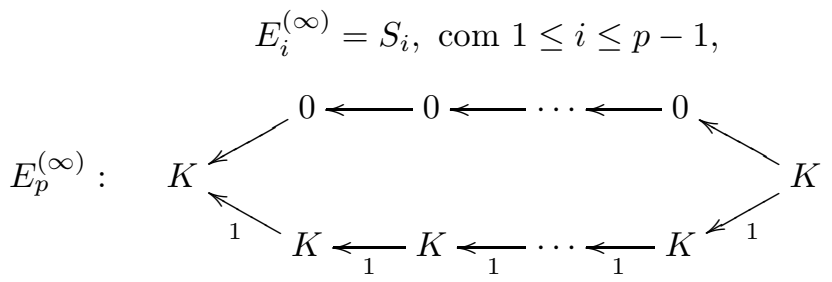

with the following properties:

$$
\tau E_{i+1}^{(\infty)}=E_{i}^{(\infty)}, \text { for } 1 \leq \underset{6}{i \leq p-1, \quad \text { and }} \quad \tau E_{1}^{(\infty)}=E_{p}^{(\infty)}
$$


(b) Simple regular representations in the tube $\mathcal{T}^{\Delta}$

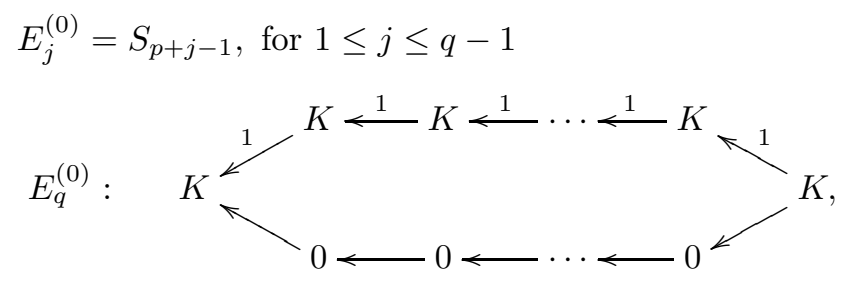

with the following properties:

$$
\tau E_{j+1}^{0}=E_{j}^{0}, \text { for } 1 \leq j \leq q-1, \quad \text { and } \quad \tau E_{1}^{0}=E_{q}^{0} .
$$

(c) Simple regular representations in the tube $\mathcal{T}_{\lambda}^{\Delta}$, with $\lambda \in K \backslash\{0\}$

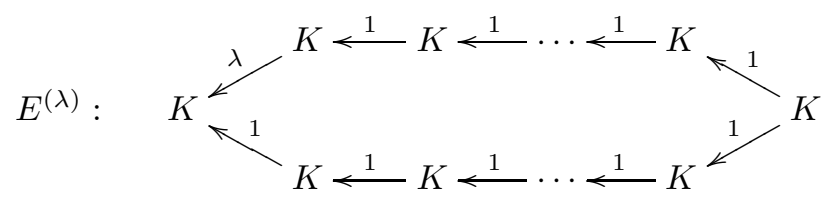

The following theorem give us information about the tubular components of $\Gamma(\bmod K \Delta)$. We will use it in order to construct the s.s. of our interest.

Theorem 6.1. (2.5 in Simsom and Skowronski, 2007, Vol. 2, Chap. XII) Assume that $A=K \Delta$. Then every component in the regular part $\mathcal{R}(A)$ of $\Gamma(\bmod A)$ is one of the following stable tubes:

(1) the tube $\mathcal{T}_{\infty}^{\Delta}$ of rank $p$ containing the $A$-modules $E_{1}^{(\infty)}, \ldots, E_{p}^{(\infty)}$.

(2) the tube $\mathcal{T}_{0}^{\Delta}$ of rank $q$ containing the $A$-modules $E_{1}^{(0)}, \ldots, E_{q}^{(0)}$.

(3) the tube $\mathcal{T}_{\lambda}^{\Delta}$ of rank 1 containing the A-modules $E^{(\lambda)}$, with $\lambda \in K \backslash\{0\}$.

Where $E_{j}^{(\infty)}, E_{i}^{(0)}$ and $E^{(\lambda)}$ are the simple regular A-modules previously defined.

We use the simple regular modules in the tubes $\mathcal{T}_{\infty}^{\Delta}$ and $\mathcal{T}_{0}^{\Delta}$ to build a s.s. of size $p+q-2$ over $K \Delta$. Let $F_{i}$ denote the module $E_{p-i}^{(\infty)}$, for $i=1, \ldots, p-1$, and let $G_{i}$ denote the module $E_{q-i}^{(0)}$, for $i=1, \ldots, q-1$. Then we have that

$$
\begin{gathered}
\tau F_{i}=F_{i+1}, \text { for } 1 \leq i \leq p-2, \tau F_{p-1}=E_{p}^{(\infty)} \text { and } \tau E_{p}=F_{1}, \\
\tau G_{i}=G_{i+1}, \text { for } 1 \leq i \leq q-2, \tau E_{q}=G_{1} \text { and } \tau E_{q}=G_{1} .
\end{gathered}
$$

The sequence $\left(F_{1}, \ldots, F_{p-1}\right)$ will be denoted by $F$ and the set $\left\{F_{1}, \ldots, F_{p-1}\right\}$ by $\mathcal{F}$. Analogously, $\left(G_{1}, \ldots, G_{q-1}\right)$ will be denoted by $G$ and $\left\{G_{1}, \ldots, G_{q-1}\right\}$ by $\mathcal{G}$. We claim that $F$ and $G$ are s.s..

Let $S$ be a set of modules and let $M$ be a module. For simplicity we write $\operatorname{Hom}_{A}(S, M)=0$ when $\operatorname{Hom}_{A}(X, M)=0$, for all $X \in S$. If $S$ is a set of regular modules, we denote by $\tau^{i}(S)$, with $i \in \mathbb{Z}, i \neq 0$, the set $\tau^{i}(S)=\left\{\tau^{i} X, X \in S\right\}$, where $\tau$ is the Auslander-Reiten translation.

Given a finite dimensional $K$-algebra, let $\left\{S_{1}, \ldots, S_{n}\right\}$ be a complete set of the isomorphism classes of simple modules, let $M$ be a module and let $\mathcal{X}$ be a set of modules. The support of $M$ is the set supp $M=$ $\left\{i \in\{1, \ldots, n\}:\left[M: S_{i}\right] \neq 0\right\}$. The support of $\mathcal{X}$ is the set Supp $\mathcal{X}=\left\{i \in\{1, \ldots, n\}:\left[M: S_{i}\right] \neq 0, M \in \mathcal{X}\right\}$.

Lemma 6.2. Let $n \in \mathbb{N}, n \neq 0$. Then:

(1) (a) If $p \mid n$, then $\operatorname{Supp} \tau^{n} \mathcal{F}=\{1, \ldots, p-1\}=\operatorname{Supp} \mathcal{F}$.

(b) If $n \equiv r(\bmod p)$ and $1 \leq r \leq p-1$, then

$$
\begin{aligned}
& \operatorname{Supp} \tau^{n} \mathcal{F}=\tau^{r} \mathcal{F}=\{0, \ldots, p+q-1\} \backslash\{p-r\} \\
& \operatorname{Supp} \tau^{-n} \mathcal{F}=\tau^{-r} \mathcal{F}=\{0, \ldots, p+q-1\} \backslash\{r\} .
\end{aligned}
$$

(2) (a) If $q \mid n$, then $\operatorname{Supp} \tau^{n} \mathcal{G}=\mathcal{G}=\{p, \ldots, p+q-2\}$.

(b) If $n \equiv r(\bmod q)$ and $1 \leq r \leq q-1$, then

$$
\begin{aligned}
\operatorname{Supp} \tau^{n} \mathcal{G}=\tau^{r} \mathcal{G} & =\{0, \ldots, p+q-1\} \backslash\{p+q-r-1\} \\
\operatorname{Supp} \tau^{-n} \mathcal{G}=\tau^{-r} \mathcal{G} & =\{0, \ldots, p+q-1\} \backslash\{p+r-1\} .
\end{aligned}
$$


Lemma 6.3. Given a hereditary algebra.

(1) If $P_{j}$ and $P_{m}$ are projective indecomposable modules then $\operatorname{Ext}^{1}\left(\tau^{-t} P_{j}, \tau^{-t-r} P_{m}\right)=0$, for all $t, r \geq 0$.

(2) If $I_{j}$ and $I_{m}$ are injective indecomposable A-modules then $\operatorname{Ext}^{1}\left(\tau^{t} I_{j}, \tau^{t-r} I_{m}\right)=0$, for all $t \geq r \geq 0$.

Proof. We just prove the statement (1) because the proof of (2) is similar. By the Auslander formula we have that $\operatorname{Ext}^{1}\left(\tau^{-t} P_{j}, \tau^{-t-r} P_{m}\right) \cong D \operatorname{Hom}\left(\tau^{-r-1} P_{m}, P_{j}\right)$. But $\operatorname{Hom}\left(\tau^{-r-1} P_{m}, P_{j}\right)=0$, otherwise $P_{j}$ would have a non-projective predecessor in $\Gamma(\bmod A)$.

Proposition 6.4. If $Y$ is a posprojective A-module such that $(F, G, Y)$ is a s.s., then $Y$ is one of the modules in the following list:

(1) $P_{0}$.

(2) $P_{p+q-1}$.

(3) $\tau^{-t} P_{0}$, with $t \geq 1$ such that $p \mid t$ and $q \mid t$.

(4) $\tau^{-t} P_{p+q-1}$, with $t \geq 1$ such that $p \mid t$ and $q \mid t$.

(5) $\tau^{-t} P_{p-r}$, with $t \geq 1$ such that $q \mid t, t \equiv r(\bmod p)$ and $1 \leq r \leq p-1$.

(6) $\tau^{-t} P_{p+q-r-1}$, with $t \geq 1$ such that $p \mid t, t \equiv r(\bmod q)$ and $1 \leq r \leq q-1$.

Proof. If $M$ is a regular module, then $\operatorname{Ext}_{A}^{1}\left(\tau^{-t} P_{j}, M\right) \cong D \operatorname{Hom}_{A}\left(\tau^{-1} M, \tau^{-t} P_{j}\right)=0$, for $t \geq 0$. Because of that, to prove that $\left(F, G, \tau^{-t} P_{j}\right)$ is a s.s. we need to show that $\operatorname{Hom}_{A}\left(\tau^{-t} P_{j}, \mathcal{F}\right)=0=\operatorname{Hom}_{A}\left(\tau^{-t} P_{j}, \mathcal{G}\right)$. On the other hand, as a consequence of Auslander formula, we have that

$$
\operatorname{Hom}_{A}\left(\tau^{-t} P_{j}, \mathcal{F}\right) \cong \operatorname{Hom}_{A}\left(P_{j}, \tau^{t} \mathcal{F}\right) \text { and } \operatorname{Hom}_{A}\left(\tau^{-t} P_{j}, \mathcal{G}\right) \cong \operatorname{Hom}_{A}\left(P_{j}, \tau^{t} \mathcal{G}\right) .
$$

Therefore,

$$
\begin{gathered}
\operatorname{Hom}_{A}\left(P_{j}, \tau^{t} \mathcal{F}\right)=0 \text { if, and only if, } j \notin \operatorname{Supp} \tau^{t} \mathcal{F} \text { and } \\
\operatorname{Hom}_{A}\left(P_{j}, \tau^{t} \mathcal{G}\right)=0 \text { if, and only if, } j \notin \operatorname{Supp} \tau^{t} \mathcal{G} .
\end{gathered}
$$

It follows that $\left(F, G, \tau^{-t} P_{j}\right)$ is a s.s. if, and only if, $j \in\left(\operatorname{Supp} \tau^{t} \mathcal{G}\right)^{\prime} \cap\left(\operatorname{Supp} \tau^{t} \mathcal{F}\right)^{\prime}$, where $\left(\operatorname{Supp} \tau^{t} \mathcal{G}\right)^{\prime}$ denotes the complement of the set $\left(\operatorname{Supp} \tau^{t} \mathcal{G}\right)$ with relation to the set $\{0, \ldots, p+q-1\}$.

Case 1. If $t=0$, then $Y \cong P_{j}$ and $\left(F, G, P_{j}\right)$ is a s.s. if, and only if, $j \in(\operatorname{Supp} \mathcal{F})^{\prime} \cap(\operatorname{Supp} \mathcal{G})^{\prime}=\{0, p+q-1\}$. Case 2. If $t$ is such that $p \mid t$ e $q \mid t$ then, according to 6.2 Supp $\tau^{t} \mathcal{F}=\operatorname{Supp} \mathcal{F}$ and $\operatorname{Supp} \tau^{t} \mathcal{G}=\operatorname{Supp} \mathcal{G}$. Therefore, $\left(F, G, \tau^{-t} P_{j}\right)$ is a s.s if, and only if, $j \in\{0, p+q-1\}$.

Case 3. If $t$ is such that $q \mid t$ and $t \equiv r(\bmod p)$, with $1 \leq r \leq p-1$, then by 6.2 we have that $\left(\operatorname{Supp} \tau^{t} \mathcal{F}\right)^{\prime}=$ $\{p-r\}$ and $\left(\operatorname{Supp} \tau^{t} \mathcal{G}\right)^{\prime}=\{0, \ldots, p-1\} \cup\{p+q-1\}$. Therefore, in this case, $\left(F, G, \tau^{-t} P_{j}\right)$ is a s.s. if, and only if, $j=p-r$.

Case 4. If $t$ is such that $p \mid t$ and $q \not t$, then similarly to the previous case we conclude that $\left(F, G, \tau^{-t} P_{j}\right)$ is a s.s. if, and only if, $j=p+q-r-1$, where $r$ is such that $t \equiv r(\bmod q)$ and $1 \leq r \leq q-1$.

Case 5. If $t \equiv r_{1}(\bmod p)$ and $t \equiv r_{2}(\bmod q)$, with $0<r_{1}<p$ and $0<r_{2}<q$ then $\left(\operatorname{Supp} \tau^{r_{1}} \mathcal{F}\right)^{\prime} \cap$ $\left(\operatorname{Supp} \tau^{r_{2}} \mathcal{G}\right)^{\prime}=\left\{p-r_{1}\right\} \cap\left\{p+q-r_{2}-1\right\}=\phi$. Therefore there is no a s.s. with these conditions for $t$.

Proposition 6.5. If $Y$ is a preinjective A-module such that $(F, G, Y)$ is a s.s., then $Y$ is one of the modules in the following list

(1) $\tau^{t} I_{p}$, with $t \geq 1$ such that $t \equiv p-1(\bmod p)$ and $q \mid t$.

(2) $\tau^{t} I_{1}$, with $t \geq 1$ such that $t \equiv q-1(\bmod q)$ and $p \mid t$.

(3) $\tau^{t} I_{0}$, with $t \geq 1$ such that $t \equiv p-1(\bmod p)$ and $t \equiv q-1(\bmod q)$.

(4) $\tau^{t} I_{p+q-1}$, with $t \geq 1$ such that $t \equiv p-1(\bmod p)$ and $t \equiv q-1(\bmod q)$.

(5) $\tau^{t} I_{r+1}$, with $t \geq 1$ such that $t \equiv r(\bmod p), r \neq p-1$ and $t \equiv q-1(\bmod q)$.

(6) $\tau^{t} I_{p+r}$, with $t \geq 1$ such that $t \equiv r(\bmod q), r \neq q-1$ and $t \equiv p-1(\bmod p)$.

Proof. Let $Y$ be a preinjective $A$-module and let $M$ be a regular $A$-module. Then $Y \cong \tau^{k} I_{j}$, for $k \geq 0$ and $j \in\{0,1, \ldots, p, \ldots, p+q-1\}$. Hence $\operatorname{Hom}_{A}(Y, M)=0$. On the other hand, by the Auslander formula, we have that $\operatorname{Ext}_{A}^{1}\left(\tau^{k} I_{j}, M\right) \cong D \operatorname{Hom}_{A}\left(\tau^{-1} M, \tau^{k} I_{j}\right) \cong D \operatorname{Hom}_{A}\left(\tau^{-(k+1)} M, I_{j}\right)$. It follows that $\operatorname{Ext}_{A}^{1}\left(\tau^{k} I_{j}, M\right)=0$ if, and only if, $j \notin \operatorname{supp} \tau^{-(k+1)} M$. Therefore we conclude that

$$
\left(F, G, \tau^{k} I_{j}\right) \text { is a s.s. if, and only if, } j \in\left(\operatorname{Supp} \tau^{-(k+1)} \mathcal{F}\right)^{\prime} \cap\left(\operatorname{Supp} \tau^{-(k+1)} \mathcal{G}\right)^{\prime} .
$$

We consider several cases in order to find all the s.s. of the form $(F, G, Y)$ with $Y$ preinjective. 
Case 1. Let $Y \cong I_{j}$ with $j \in\{0,1, \ldots, p, \ldots, p+q-1\}$. Then, by 6.2 we have that $\left(\operatorname{Supp} \tau^{-1} \mathcal{F}\right)^{\prime} \cap$ $\left(\operatorname{Supp} \tau^{-1} \mathcal{G}\right)^{\prime}=\{1\} \cap\{p\}=\phi$. Therefore there is no $j$ such that $\left(F, G, I_{j}\right)$ is a s.s..

Case 2. Let $Y \cong \tau^{t} I_{j}$, with $t \geq 1$ such that $p \mid t$ and $q \mid t$. Then

$$
\left(\operatorname{Supp} \tau^{-(t+1)} \mathcal{F}\right)^{\prime} \cap\left(\operatorname{Supp} \tau^{-(t+1)} \mathcal{G}\right)^{\prime}=\left(\operatorname{Supp} \tau^{-1} \mathcal{F}\right)^{\prime} \cap\left(\operatorname{Supp} \tau^{-1} \mathcal{G}\right)^{\prime}=\phi .
$$

Case 3. Let $Y \cong \tau^{t} I_{j}$, with $t$ such that $p \not t$ and $q \mid t$. Let $t \equiv r(\bmod p), 1 \leq r \leq p-1$. Here we need to consider two cases: when $r=p-1$ and when $r \neq p-1$.

- If $r=p-1$, then using 6.2 we have that

$$
\begin{gathered}
\operatorname{Supp} \tau^{-(t+1)} \mathcal{F}=\operatorname{Supp} \tau^{-(r+1)} \mathcal{F}=\operatorname{Supp} \tau^{-p} \mathcal{F}=\operatorname{Supp} \mathcal{F} \text { and } \\
\operatorname{Supp} \tau^{-(t+1)} \mathcal{G}=\operatorname{Supp} \tau^{-1} \mathcal{G}=\{0, \ldots, p+q-1\} \backslash\{p\} .
\end{gathered}
$$

Hence $\left(\operatorname{Supp} \tau^{-(t+1)} \mathcal{F}\right)^{\prime} \cap \operatorname{Supp}\left(\tau^{-(t+1)} \mathcal{G}\right)^{\prime}=\{p\}$ and therefore $\left(F, G, \tau^{t} I_{p}\right)$ is a s.s..

- If $r \neq p-1$, we have that

$$
\operatorname{Supp} \tau^{-(t+1)} \mathcal{F}=\operatorname{Supp} \tau^{-(r+1)} \mathcal{F}=\{0, \ldots, p+q-1\} \backslash\{r+1\} .
$$

Then $\left(\operatorname{Supp} \tau^{-(t+1)} \mathcal{F}\right)^{\prime} \cap\left(\operatorname{Supp} \tau^{-(t+1)} \mathcal{G}\right)^{\prime}=\{r+1\} \cap\{p\}=\phi$. Hence there is no a $j$ such that $\left(F, G, \tau^{t} I_{j}\right)$ is a s.s.

Case 4. Let $Y \cong \tau^{t} I_{j}$ with $t \geq 1$ such that $q \backslash t$ and $p \mid t$. Let $r \equiv t(\bmod q)$, with $1 \leq r \leq q-1$. Here we need to consider two cases: when $r=q-1$ and when $r \neq q-1$.

- If $r=q-1$, then

$$
\begin{aligned}
\operatorname{Supp} \tau^{-(t+1)} \mathcal{G}=\operatorname{Supp} \tau^{-(r+1)} \mathcal{G} & =\operatorname{Supp} \tau^{-q} \mathcal{G}=\operatorname{Supp} \mathcal{G} . \text { and } \\
\operatorname{Supp} \tau^{-(t+1)} \mathcal{F}=\operatorname{Supp} \tau^{-1} \mathcal{F} & =\{0, \ldots, p+q-1\} \backslash\{1\}
\end{aligned}
$$

It follows that $\left(\operatorname{Supp} \tau^{-(t+1)} \mathcal{F}\right)^{\prime} \cap\left(\operatorname{Supp} \tau^{-(t+1)} \mathcal{G}\right)^{\prime}=\{1\}$. Therefore $\left(F, G, \tau^{t} I_{1}\right)$ is a s.s.

- If $r \neq q-1$, we have that

$$
\operatorname{Supp} \tau^{-(t+1)} \mathcal{G}=\operatorname{Supp} \tau^{-(r+1)} \mathcal{G}=\{0, \ldots p+q-1\} \backslash\{p+r\} .
$$

Then $\left(\operatorname{Supp} \tau^{-(t+1)} \mathcal{F}\right)^{\prime} \cap\left(\operatorname{Supp} \tau^{-(t+1)} \mathcal{G}\right)^{\prime}=\{1\} \cap\{p+r\}=\phi$ and therefore there is no a $j$ such that $\left(F, G, \tau^{t} I_{j}\right)$ is a s.s.

Case 5. Let $Y \cong \tau^{t} I_{j}$, with $t$ such that $q \backslash t$ and $p \nmid t$. Let $r_{1}$ and $r_{2}$ such that $t \equiv r_{1}(\bmod p), t \equiv r_{2}(\bmod q)$, $1 \leq r_{1} \leq p-1$ and $1 \leq r_{2} \leq q-1$. We consider several cases:

- Let $r_{1}=p-1$ and let $r_{2}=q-1$. We have that

$$
\left(\operatorname{Supp} \tau^{-(t+1)} \mathcal{F}\right)^{\prime} \cap\left(\operatorname{Supp} \tau^{-(t+1)} \mathcal{G}\right)^{\prime}=(\operatorname{Supp} \mathcal{F})^{\prime} \cap(\operatorname{Supp} \mathcal{G})^{\prime}=\{0, p+q-1\} .
$$

Therefore $\left(F, G, \tau^{t} I_{0}\right)$ and $\left(F, G, \tau^{t} I_{p+q-1}\right)$ are s.s..

- Let $r_{2}=q-1$ and let $r_{1} \neq p-1$. Then

$$
\begin{gathered}
\operatorname{Supp} \tau^{-(t+1)} \mathcal{G}=\operatorname{Supp} \mathcal{G} \text { and } \\
\operatorname{Supp} \tau^{-(t+1)} \mathcal{F}=\operatorname{Supp} \tau^{-\left(r_{1}+1\right)} \mathcal{F}=\{0, \ldots, p+q-1\} \backslash\left\{r_{1}+1\right\} .
\end{gathered}
$$

Hence $\left(F, G, \tau^{t} I_{r_{1}+1}\right)$ is a s.s..

- Let $r_{1}=p-1$ and let $r_{2} \neq q-1$. In this case we have that

$$
\begin{gathered}
\operatorname{Supp} \tau^{-(t+1)} \mathcal{F}=\operatorname{Supp} \mathcal{F} \text { and } \\
\operatorname{Supp} \tau^{-(t+1)} \mathcal{G}=\operatorname{Supp} \tau^{-\left(r_{2}+1\right)} \mathcal{G}=\{0, \ldots p+q-1\} \backslash\left\{p+r_{2}\right\} .
\end{gathered}
$$

Then $\left(F, G, \tau^{t} I_{p+r_{2}}\right)$ is a s.s..

- If $r_{1} \neq p-1$ and $r_{2} \neq q-1$, then

$$
\begin{aligned}
& \operatorname{Supp} \tau^{-(t+1)} \mathcal{G}=\operatorname{Supp} \tau^{-\left(r_{2}+1\right)} \mathcal{G}=\{0, \ldots p+q-1\} \backslash\left\{p+r_{2}\right\} \text { e } \\
& \operatorname{Supp} \tau^{-(t+1)} \mathcal{F}=\operatorname{Supp} \tau^{-\left(r_{1}+1\right)} \mathcal{F}=\{0, \ldots, p+q-1\} \backslash\left\{r_{1}+1\right\}
\end{aligned}
$$

Consequently, there is no a s.s. in this case.

The following result is well known and it is not difficult to prove. 
Lemma 6.6. Given a finite dimensional $K$-algebra $B$ and

$$
0 \longrightarrow L \stackrel{\left(f^{\prime} g^{\prime}\right)^{t}}{\longrightarrow} X \oplus \stackrel{(f g)}{\longrightarrow} M \longrightarrow 0
$$

an almost split sequence in $\bmod B$. Then

(1) If $f^{\prime}$ is a monomorphism (resp. epimorphism), then $g$ is a monomorphism (resp. epimorphism).

(2) If $g^{\prime}$ is a monomorphism (resp. epimorphism), then $f$ is a monomorphism (resp. epimorphism).

Proposition 6.7. Let $A=K \Delta$. The postprojective component $\mathcal{P}(A)$ of $\Gamma(\bmod A)$ has the following properties:

(1) Any irreducible morphism $W \longrightarrow V$ between indecomposable modules in $\mathcal{P}(A)$ is a monomorphism.

(2) The module $P_{p+q-1}$ and all their sucessors in $\mathcal{P}(A)$ are sincere.

(3) The integer $p$ is the minimal with the property that the modules $\tau^{-r} P_{i}$ with $i \in\{0, \ldots, p+q-1\}$ are sincere.

(4) If $0 \leq i \leq p-1$, then

- The smallest integer $r$ such that $\tau^{-r} P_{i}$ is sincere is $r=p-i$. Furthermore, all the A-modules of the form $\tau^{-k} P_{i}$, with $k>p-i$, are sincere.

- If $0<k<p-i$, then the composition factors of $\tau^{-k} P_{i}$ are $S_{j}$ with $0 \leq j \leq i+k$ and $p \leq j \leq p+k-1$.

- The composition factors of $P_{i}$ are $S_{j}$ with $0 \leq j \leq i$.

(5) If $p \leq i \leq p+q-2$, then

- If $i \leq q-1$, then the smallest integer $r$ such that $\tau^{-r} P_{i}$ is sincere is $r=p$. Furthermore, all the $A$-modules in the form $\tau^{-k} P_{i}$, with $k>p$, are sincere.

- If $q-1 \leq i \leq p+q-1$, then the smallest integer $r$ such that $\tau^{-r} P_{i}$ is sincere is $r=p+q-1-i$. Furthermore, all the $A$-modules of the form $\tau^{-k} P_{i}$, with $k>p+q-1-i$, are sincere.

- If $\tau^{-k} P_{i}$ is not sincere, then their composition factors are $S_{j}$ with $p \leq j \leq i+k$ and $0 \leq j \leq k$.

(6) If $1 \leq k \leq p-1$, then the composition factors of $\tau^{-k} P_{0}$ are $S_{j}$ with $0 \leq j \leq k$ and $p \leq j \leq p-1+k$.

Proof. Our proof use the structure of the postprojective component $\mathcal{P}(A)$ of $\Gamma(\bmod A)$ which looks as follows

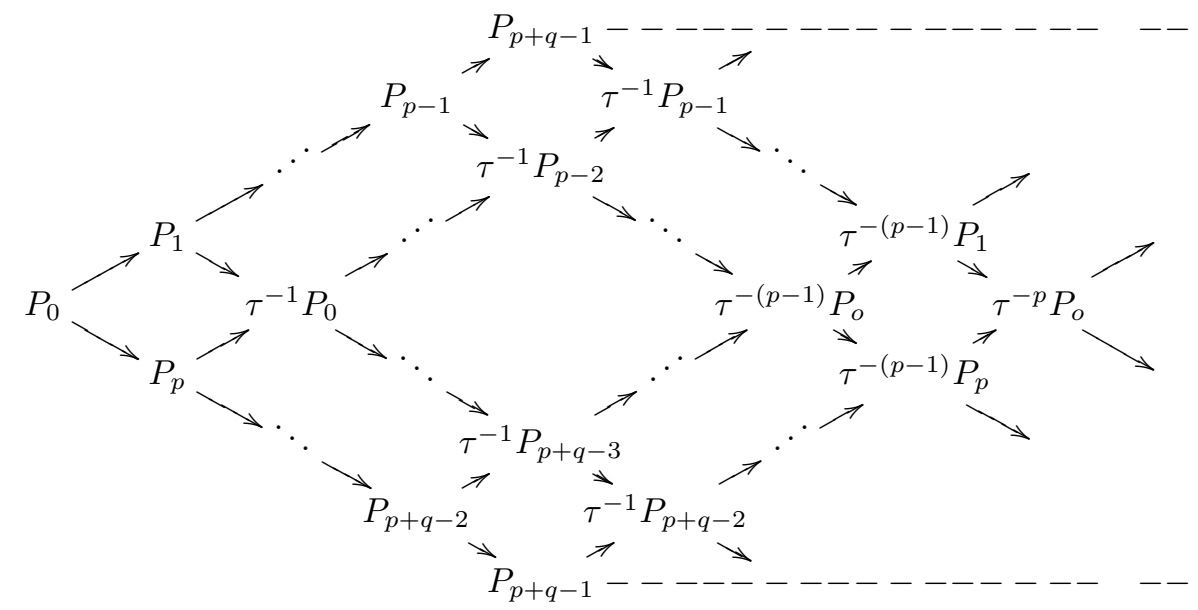

In order to prove (1) we observe that in the almost split sequence

$$
0 \longrightarrow P_{0} \stackrel{\left(\alpha^{\prime} \beta^{\prime}\right)^{t}}{\longrightarrow} P_{1} \oplus P_{p} \stackrel{(\alpha \beta)}{\longrightarrow} \tau^{-1} P_{0} \longrightarrow 0,
$$

the morphisms $\alpha^{\prime}$ and $\beta^{\prime}$ are mono, because are irreducible morphisms betwen projective modules. Then, by 6.6, $\alpha$ and $\beta$ are mono too. Analogously, in all almost split sequences begining in a projective module the arrows represent monomorphisms. Hence, according to the shape $\mathcal{P}(A)$, all the arrows in this component represent monomorphisms.

Now we prove (2). We note that in $\mathcal{P}(A)$ there are paths

$$
P_{0} \rightarrow P_{1} \rightarrow \cdots \rightarrow P_{p-1} \rightarrow P_{p+q-1} \text { and } P_{0} \longrightarrow P_{p} \rightarrow \cdots \rightarrow P_{p+q-1} \rightarrow P_{p+q-1},
$$


where, by (1), all the arrows represent monomorphisms. Therefore, $P_{p+q-1}$ and all their sucessors are sincere modules.

For (3), we observe that none of the predecessors in $\mathcal{P}(A)$ of modules in the paths below

$$
\begin{gathered}
P_{p+q-1} \longrightarrow \tau^{-1} P_{p-1} \longrightarrow \tau^{-2} P_{p-2} \longrightarrow \cdots \longrightarrow \tau^{-(p-1)} P_{0} \longrightarrow \tau^{-p} P_{0} \\
P_{p+q-1} \longrightarrow \tau^{-1} P_{p+q-2} \longrightarrow \tau^{-2} P_{p+q-3} \longrightarrow \cdots \longrightarrow \tau^{-(p-1)} P_{p} \longrightarrow \tau^{-p} P_{0}
\end{gathered}
$$

have $S_{p+q-1}$ as composition factor. In particular, $\tau^{-(p-1)} P_{0}$ don't have $S_{p+q-1}$ as composition factor. Therefote, by (1), none of their predecessors have $S_{p+q-1}$ as composition factor. On the other hand, any successor of $\tau^{-(p-1)} P_{0}$, and any of modules in (6.3) and (6.4), are sincere.

In order to show (4), we note that all the modules $\tau^{-(p-i)} P_{i}$, with $0 \leq i \leq p-1$, are in the path (6.3) and therefore are sincere. Moreover, as stated earlier, any predecessor of some of the modules which appear in (6.3) have no $S_{p+q-1}$ as composition factor. It follows that, if $0 \leq i \leq p-1$ then none of the modules of the form $\tau^{-k} P_{i}$, with $k<p-i$, are sincere.

On the other hand, the predeccessors of $P_{i}$, with $0 \leq i \leq p-1$, are the projectives $P_{0}, P_{1}, \ldots, P_{i-1}$. Thus the compostion factors of $P_{i}$, with $0 \leq i \leq p-1$, are $S_{j}$ with $0 \leq j \leq i$. Analogously, for $p \leq i<p+q-1$, the compostion factors of $P_{i}$ are $S_{j}$, with $p \leq j \leq i$ and $j=0$.

Let $0 \leq i \leq p-1$. Then in $\mathcal{P}(A)$ there are paths

$$
\begin{aligned}
P_{i+k} & \rightarrow \tau^{-1} P_{i+k-1} \rightarrow \tau^{-2} P_{i+k-2} \rightarrow \cdots \rightarrow \tau^{-(k-1)} P_{i+1} \rightarrow \tau^{-k} P_{i} \\
P_{p+k-1} & \rightarrow \tau^{-1} P_{p+k-2} \rightarrow \tau^{-2} P_{p+k-3} \rightarrow \cdots \rightarrow \tau^{-(k-1)} P_{p} \rightarrow \tau^{-k} P_{0} \rightarrow \\
\tau^{-k} P_{1} & \rightarrow \tau^{-k} P_{2} \rightarrow \cdots \rightarrow \tau^{-k} P_{i}
\end{aligned}
$$

Furthermore, there are no paths between $P_{j}$, with $j \geq i+k$, and $\tau^{-k} P_{i}$. Hence the composition factors of $\tau^{-k} P_{i}$, up to multiplicity, are the composition factors of $P_{i+k}$ and of $P_{p+k-1}$. In other words, the composition factors of $\tau^{-k} P_{i}$ are $S_{j}$ with $0 \leq j \leq i$ and $p \leq i<p+q-1$.

The proof of (5), is analogous to the previous and part (6) is left to the reader, since it follows a similar argument.

Proposition 6.8. Let $A=K \Delta$. The complete list of s.s. $(X, F, G, Y)$, where $Y$ is a postprojective A-module, is as follows:

(1) $\left(S_{p+q-1}, F, G, P_{0}\right)$.

(2) $\left(X, F, G, P_{p+q-1}\right)$, where

$$
X \cong\left\{\begin{array}{l}
\tau^{-p+1} P_{0}, \text { if } p=q \\
\tau^{-p+1} P_{q-1}, \text { if } p \neq q
\end{array}\right.
$$

(3) $\left(X, F, G, \tau^{-t} P_{p+q-1}\right)$ with $t \in \mathbb{N}$ such that $p \mid t$ and $q \mid t$, where

$$
X \cong\left\{\begin{array}{l}
\tau^{-t-p+1} P_{0}, \text { if } p=q \\
\tau^{-t-p+1} P_{q-1}, \text { if } p \neq q
\end{array}\right.
$$

(4) $\left(\tau^{-t+1} P_{p+q-1}, F, G, \tau^{-t} P_{0}\right)$, with $t \in \mathbb{N}$ such that $p \mid t$ and $q \mid t$.

(5) $\left(\tau^{-t-(p-r-1)} P_{q+r-1}, F, G, \tau^{-t} P_{p-r}\right)$, with $t, r \in \mathbb{N}$ such that $q \mid t, t \equiv r(\bmod p)$ and $1 \leq r \leq p-1$.

(6) $\left(X, F, G, \tau^{-t} P_{p+q-r-1}\right)$, with $t, r \in \mathbb{N}$ such that $p \mid t, t \equiv r(\bmod q)$ and $1 \leq r \leq q-1$, where

$$
X \cong\left\{\begin{array}{l}
\tau^{-t-q+r+1} P_{p-q+r}, \text { if } p \leq q-r \\
\tau^{-t-p+1} P_{q-r-1}, \text { if } p>q-r
\end{array}\right.
$$

Proof. The Proposition 6.4 characterized s.s. of the form $(F, G, Y)$, with $Y$ a postprojective $A$-module. By using this characterization we obtain stratifying systems of type $(X, F, G, Y)$.

The quiver $\Delta$ has $p+q$ vértices then, by 5.1 and 5.2, the module $X$ such that $\left(X, F, G, \tau^{-t} P_{l}\right)$ is a c.s.s. is unique. Thus, the proof consist in to verify that the indecomposable module $X$ satisfies the following conditions:

- $\operatorname{Ext}_{A}^{1}(X, X)=0$.

- $\operatorname{Hom}_{A}(\mathcal{F}, X)=0$ and $\operatorname{Hom}_{A}(\mathcal{G}, X)=0$.

- $\operatorname{Ext}_{A}^{1}(\mathcal{F}, X)=0$ and $\operatorname{Ext}_{A}^{1}(\mathcal{G}, X)=0$.

- $\operatorname{Hom}_{A}\left(\tau^{-t} P_{l}, X\right)=0$ and $\operatorname{Ext}_{A}^{1}\left(\tau^{-t} P_{l}, X\right)=0$. 
We observe that all the modules $X$ considered in this proof, except $I_{p+q-1}$, are of the form $X \cong \tau^{-k} P_{j}$, with $k \geq 0$, and therefore are indecomposable and have no self-extensions. Moreover, there is no nonzero morphism from a regular module to a posprojective module, that is, if $R$ is a regular module then $\operatorname{Hom}_{A}\left(R, \tau^{-k} P_{j}\right)=0$. On the other hand, by the Auslander formula, we have that

$$
\operatorname{Ext}_{A}^{1}\left(R, \tau^{-k} P_{j}\right) \cong D \operatorname{Hom}_{A}\left(\tau^{-k} P_{j}, \tau R\right) \cong D \operatorname{Hom}_{A}\left(P_{j}, \tau^{k+1} R\right) \text {. }
$$

Therefore we have that $\operatorname{Ext}_{A}^{1}\left(\mathcal{F}, \tau^{-k} P_{j}\right)=0$ and $\operatorname{Ext}_{A}^{1}\left(\mathcal{G}, \tau^{-k} P_{j}\right)=0$ if, and only if, $j \in\left(\operatorname{Supp} \tau^{k+1} \mathcal{F}\right)^{\prime} \cap$ $\left(\operatorname{Supp} \tau^{k+1} \mathcal{G}\right)^{\prime}$. In view of these observations, if $\left(F, G, \tau^{-t} P_{l}\right)$ is a s.s. and $X \cong \tau^{-k} P_{j}$, in order to show that $\left(X, F, G, \tau^{-t} P_{l}\right)$ is a s.s. is sufficient to check the following conditions:

- $j \in\left(\operatorname{Supp} \tau^{k+1} \mathcal{F}\right)^{\prime} \cap\left(\operatorname{Supp} \tau^{k+1} \mathcal{G}\right)^{\prime}$.

- $\operatorname{Hom}_{A}\left(\tau^{-t} P_{l}, X\right)=0$ (ou equivalently $\left[\tau^{t} X: S_{l}\right]=0$ ).

- $\operatorname{Ext}_{A}^{1}\left(\tau^{-t} P_{l}, X\right)=0$.

In what follows we find these conditions for each of the sequences stated in the proposition. (1) First let us check that $\left(S_{p+q-1}, F, G, P_{0}\right)$ is a s.s.. As the vertice $p+q-1$ is a source then the simple $A$-module $S_{p+q-1}$ is injective and thus

$$
\operatorname{Ext}_{A}^{1}\left(\mathcal{F}, S_{p+q-1}\right)=0, \operatorname{Ext}_{A}^{1}\left(\mathcal{G}, S_{p+q-1}\right)=0 \text { and } \operatorname{Ext}_{A}^{1}\left(P_{0}, S_{p+q-1}\right)=0 .
$$

On the other hand, since $p+q-1 \notin(\operatorname{Supp} \mathcal{F} \cup \operatorname{Supp} \mathcal{G})$ and $S_{p+q-1} \cong I_{p+q-1}$ then

$$
\operatorname{Hom}_{A}\left(\mathcal{F}, S_{p+q-1}\right)=0, \operatorname{Hom}_{A}\left(\mathcal{G}, S_{p+q-1}\right)=0 \text { and } \operatorname{Hom}_{A}\left(P_{0}, S_{p+q-1}\right)=0 .
$$

(2) To complete the s.s. $\left(F, G, P_{p+q-1}\right)$ we have to consider two cases:

- If $p=q$ and $X \cong \tau^{-p+1} P_{0}$, then by 6.2 we have that $\left(\operatorname{Supp} \tau^{p} \mathcal{F}\right)^{\prime} \cap\left(\operatorname{Supp} \tau^{p} \mathcal{G}\right)^{\prime}=\{0, p+q-1\}$. On the other hand $\left[\tau^{-p+1} P_{0}: S_{p+q-1}\right]=0$, by 6.7 (6). Therefore the sequence $\left(\tau^{-p+1} P_{0}, F, G, P_{p+q-1}\right)$ is a s.s..

- If $p \neq q$ and $X=\tau^{-p+1} P_{q-1}$ then we have that $\left(\operatorname{Supp} \tau^{p} \mathcal{F}\right)^{\prime} \cap\left(\operatorname{Supp} \tau^{p} \mathcal{G}\right)^{\prime}=\{q-1\}$ and, by 6.7 (5), we have that $\left[\tau^{-p+1} P_{q-1}: S_{p+q-1}\right]=0$.

In both cases we have the item 2 of the list.

(3) In order to complete the s.s. $\left(F, G, \tau^{-t} P_{p+q-1}\right)$, with $t \geq 1$ such that $p \mid t$ and $q \mid t$, we consider the following two cases:

- Suppose $p=q$. We claim that the sequence $\left(\tau^{-t-p+1} P_{0}, F, G, \tau^{-t} P_{p+q-1}\right)$, is a s.s.. In fact, $\left(\operatorname{Supp} \tau^{t+p} \mathcal{F}\right)^{\prime} \cap\left(\operatorname{Supp} \tau^{t+p} \mathcal{G}\right)^{\prime}=\{0, p+q-1\}$.

On the other hand, by 6.7 (6), $\operatorname{Hom}_{A}\left(\tau^{-t} P_{p+q-1}, \tau^{-t-p+1} P_{0}\right) \cong 0$. Moreover, by 6.3 (1),

$\operatorname{Ext}_{A}^{1}\left(\tau^{-t} P_{0}, \tau^{-t-p+1} P_{q-1}\right)=0$. These conditions complete the verification of our claim.

- Suppose $p \neq q$. In this case we see that $\left(\tau^{-t-p+1} P_{q-1}, F, G, \tau^{-t} P_{p+q-1}\right)$ is a c.s.s. Indeed, $\left(\operatorname{Supp} \tau^{t+p} \mathcal{F}\right)^{\prime} \cap$

$\left(\operatorname{Supp} \tau^{t+p} \mathcal{G}\right)^{\prime}=\{q-1\}$ by [6.2, $\left[\tau^{-p+1} P_{q-1}: S_{p+q-1}\right]=0$ by 6.7 (5) and finally by 6.3 (1) $\operatorname{Ext}_{A}^{1}\left(\tau^{-t} P_{p+q-1}, \tau^{-t-p+1} P_{q-1}\right)=0$.

(4) We show that $\left(\tau^{-t+1} P_{p+q-1}, F, G, \tau^{-t} P_{0}\right)$, with $t \geq 1$ such that $p \mid t$ and $q \mid t$ is a c.s.s.. In fact, $\left(\operatorname{Supp} \tau^{t} \mathcal{F}\right)^{\prime} \cap$ $\left(\operatorname{Supp} \tau^{t} \mathcal{G}\right)^{\prime}=\{0, p+q-1\}$. On the other hand, by using the Auslander formula we have

$$
\operatorname{Hom}_{A}\left(\tau^{-t} P_{0}, \tau^{-t+1} P_{p+q-1}\right) \cong D \operatorname{Ext}_{A}^{1}\left(P_{p+q-1}, \tau^{-1} P_{0}\right) \cong 0
$$

and

$$
\operatorname{Ext}_{A}^{1}\left(\tau^{-t} P_{0}, \tau^{-t+1} P_{p+q-1}\right) \cong D \operatorname{Hom}_{A}\left(P_{p+q-1}, P_{0}\right) \cong 0 .
$$

Then the conditions for the s.s. are verified.

(5) We prove that $\left(\tau^{-t-(p-r-1)} P_{q+r-1}, F, G, \tau^{-t} P_{p-r}\right)$, with $t, r \in \mathbb{N}$ such that $q \mid t, t \equiv r(\bmod p)$ and $1 \leq r \leq p-1$, is a s.s.. According to 6.2, we have

$$
\left(\operatorname{Supp} \tau^{t+(p-r)} \mathcal{F}\right)^{\prime} \cap\left(\operatorname{Supp} \tau^{t+(p-r)} \mathcal{G}\right)^{\prime}=(\operatorname{Supp} \mathcal{F})^{\prime} \cap\left(\operatorname{Supp} \tau^{p-r} \mathcal{G}\right)^{\prime}=\{q+r-1\} .
$$
have

Applying 6.3 (1) we can assert that $\operatorname{Ext}_{A}^{1}\left(\tau^{-t} P_{p-r}, \tau^{-t-(p-r-1)} P_{q+r-1}\right)=0$. And finally, by 6.7 (7), we

$$
\operatorname{Hom}_{A}\left(\tau^{-t} P_{p-r}, \tau^{-t-(p-r-1)} P_{q+r-1}\right) \cong \operatorname{Hom}_{A}\left(P_{p-r}, \tau^{-(p-r-1)} P_{q+r-1}\right) \cong 0 .
$$


(6) Suppose that $t, r \in \mathbb{N}$ are such that $p \mid t, t \equiv r(\bmod q)$ and $1 \leq r \leq q-1$. Let us consider two cases:

- Assume that $p \leq q-r$. Then, by 6.2, we have that

$$
\begin{aligned}
\left(\operatorname{Supp} \tau^{t+q-r} \mathcal{F}\right)^{\prime} \cap\left(\operatorname{Supp} \tau^{t+q-r} \mathcal{G}\right)^{\prime} & =\left(\operatorname{Supp} \tau^{q-r} \mathcal{F}\right)^{\prime} \cap(\operatorname{Supp} \mathcal{G})^{\prime} \\
& =\{p-q+r\} .
\end{aligned}
$$

We note that $q-r-1 \geq 0$, then by 6.3 (1), have

$$
\operatorname{Ext}_{A}^{1}\left(\tau^{-t} P_{p+q-r-1}, \tau^{-t-q+r+1} P_{p-q+r}\right)=0 .
$$

Furthermore, by 6.7 (4) it follows that

$$
\operatorname{Hom}_{A}\left(\tau^{-t} P_{p+q-r-1}, \tau^{-t-q+r+1} P_{p-q+r}\right) \cong \operatorname{Hom}_{A}\left(P_{p+q-r-1}, \tau^{-q+r+1} P_{p-q+r}\right) \cong 0 .
$$

Thus $\left(\tau^{-t-q+r+1} P_{p-q+r}, F, G, \tau^{-t} P_{p+q-r-1}\right)$ is a c.s.s..

- Suppose that $q-r>p$. Let $l$ such that $p+l=q-r$. Therefore by 6.2 we have that

$$
\begin{aligned}
\left(\operatorname{Supp} \tau^{t+p-1} \mathcal{F}\right)^{\prime} \cap\left(\operatorname{Supp} \tau^{t+p-1} \mathcal{G}\right)^{\prime} & =\left(\operatorname{Supp} \tau^{-l} \mathcal{F}\right)^{\prime} \cap(\operatorname{Supp} \mathcal{G})^{\prime} \\
& =\{p+l-1\} \\
& =\{q-r-1\} .
\end{aligned}
$$

Now by 6.3 (1), we have that $\operatorname{Ext}_{A}^{1}\left(\tau^{-t} P_{p+q-r-1}, \tau^{-t-p-1} P_{q-r-1}\right)=0$. Finally by 6.7 (4) we have that $\left[S_{p+q-r-1}: \tau^{-p+1} P_{q-r-1}\right]=0$.

We conclude that $\left(\tau^{-t-p+1} P_{q-r-1}, F, G, \tau^{-t} P_{p+q-r-1}\right)$ is a c.s.s..

Next result is proved in an analogous way as 6.7

Proposition 6.9. Let $A=K \Delta$. The preinjective component $\mathcal{Q}(A)$ of $\Gamma(\bmod A)$ has the following properties:

(1) Any irreducible morphism $W \longrightarrow V$ between indecomposable modules in $\mathcal{Q}(A)$ is an epimorphism.

(2) The $A$-module $I_{0}$ and all their predecessors in $\mathcal{Q}(A)$ are sincere modules.

(3) The integer $p$ is minimal with the property that the modules $\tau^{r} I_{i}$, with $i \in\{0, \ldots, p+q-1\}$ are sincere.

(4) If $1 \leq i \leq p-1$, then:

- the smallest integer $r$ such that $\tau^{r} I_{i}$ is a sincere module is $r=i$. Furthermore, all the A-modules in the form $\tau^{k} I_{i}$, with $k \geq i$, are sincere A-modules.

- if $k<i$, then the composition factors of $\tau^{k} I_{i}$ are simple A-modules $S_{j}$ with $i-k \leq j \leq p-1$ and $p+q-k \leq j \leq p+q-1$.

(5) If $p \leq i<p+q-1$, then:

- if $p \leq i<q-1$, the smallest integer $r$ such that $\tau^{r} I_{i}$ is a sincere module is $r=i-p+1$. Moreover, all the A-modules of the form $\tau^{r} I_{i}$, with $r \geq i-p+1$, are sincere.

- if $q-1 \leq i \leq p+q-1$, the smallest integer $r$ such that $\tau^{r} I_{i}$ is a sincere A-module is $r=p$. Furthermore, all the $A$-modules of the form $\tau^{r} I_{i}$, with $r \geq p$ are sincere.

- if $p \leq i \leq p+q-2$ and $k$ is such that $\tau^{k} I_{i}$ is not a sincere module, then the composition factors of $\tau^{k} I_{i}$ are simple $A$-modules $S_{j}$ with $i-k \leq j \leq p+q-1$ and $p-k \leq j \leq p-1$.

(6) If $k<p$, then the composition of $\tau^{k} I_{p+q-1}$ are simple $A$-modules $S_{j}$ with $p-k \leq j \leq p-1$ and $p+q-k \leq j \leq p+q-1$.

Proposition 6.10. Let $A=K \Delta$. The complete list of s.s. $(X, F, G, Y)$, where $Y$ is a preinjective A-module is as follows:

(1) $\left(\tau^{t} I_{p-1}, F, G, \tau^{t} I_{p}\right)$, with $t \geq 1$ such that $t \equiv p-1(\bmod p)$ and $q \mid t$.

(2) $\left(\tau^{t} I_{p+q-2}, F, G, \tau^{t} I_{1}\right)$, with $t \geq 1$ such that $t \equiv q-1(\bmod q)$ and $p \mid t$.

(3) $\left(\tau^{t+1} I_{p+q-1}, F, G, \tau^{t} I_{0}\right)$, with $t \geq 1$ such that $t \equiv p-1(\bmod p)$ and $t \equiv q-1(\bmod q)$.

(4) $\left(\tau^{t-p+1} I_{q-1}, F, G, \tau^{t} I_{p+q-1}\right)$, with $t \geq 1$ such that $t \equiv p-1(\bmod p)$ and $t \equiv q-1(\bmod q)$.

(5) $\left(\tau^{t-r} I_{p+q-r-2}, F, G, \tau^{t} I_{r+1}\right)$, with $t \geq 1$ such that $t \equiv r(\bmod p), 0<r<p-1$ et $t \equiv q-1(\bmod q)$.

(6) $\left(X, F, G, \tau^{t} I_{p+r}\right)$, with $t \geq 1$ such that $t \equiv r(\bmod q), 0<r<q-1$ and $t \equiv p-1(\bmod p)$, where

$$
X \cong\left\{\begin{array}{l}
\tau^{t-r} I_{p-(r+1)}, \text { if } r<p \\
\tau^{t-(p-1)} I_{r}, \text { if } r \geq p
\end{array}\right.
$$


Proof. By 5.1 and 5.2 there is an unique $A$-module $X$ such that $\left(X, F, G, \tau^{m} I_{i}\right)$ is a s.s.. Therefore the proof consists in verify that the indecomposable module $X$ satisfies the following conditions:

- $\operatorname{Ext}_{A}^{1}(X, X)=0$.

- $\operatorname{Hom}_{A}(\mathcal{F}, X)=0$ and $\operatorname{Hom}_{A}(\mathcal{G}, X)=0$.

- $\operatorname{Ext}_{A}^{1}(\mathcal{F}, X)=0$ and $\operatorname{Ext}_{A}^{1}(\mathcal{G}, X)=0$.

- $\operatorname{Hom}_{A}\left(\tau^{t} I_{i}, X\right)=0$ and $\operatorname{Ext}_{A}^{1}\left(\tau^{t} I_{i}, X\right)=0$.

All modules $X$ that we will consider are of the form $X \cong \tau^{l} I_{j}$ and therefore are indecomposable and have no self-extensions. Moreover if $X$ is a preinjective $A$-module and $R$ is a regular $A$-module then, by using the Auslander formula, we have $\operatorname{Ext}_{A}^{1}(R, X) \cong D \operatorname{Hom}_{A}(X, \tau R) \cong 0$. On the other hand, for $l \geq 0$, we have $\operatorname{Hom}_{A}\left(R, \tau^{l} I_{j}\right) \cong \operatorname{Hom}_{A}\left(\tau^{-l} R, I_{j}\right)$. It follows that $\operatorname{Hom}_{A}\left(R, \tau^{l} I_{j}\right)=0 \Leftrightarrow j \notin \operatorname{supp} \tau^{-l} R$. Therefore we have

$$
\operatorname{Hom}_{A}\left(\mathcal{F}, \tau^{l} I_{j}\right)=0 \text { and } \operatorname{Hom}_{A}\left(\mathcal{G}, \tau^{l} I_{j}\right)=0 \Leftrightarrow j \in\left(\operatorname{Supp} \tau^{-l} \mathcal{F}\right)^{\prime} \cap\left(\operatorname{Supp} \tau^{-l} \mathcal{G}\right)^{\prime} .
$$

According to the above remarks, if $\left(F, G, \tau^{t} I_{i}\right)$ is a s.s. and $X \cong \tau^{l} I_{j}$, in order to prove that $\left(X, F, G, \tau^{t} I_{i}\right)$ is c.s.s. is sufficient to show the following conditions:
a. $j \in\left(\operatorname{Supp} \tau^{-l} \mathcal{F}\right)^{\prime} \cap\left(\operatorname{Supp} \tau^{-l} \mathcal{G}\right)^{\prime}$
b. $\operatorname{Hom}_{A}\left(\tau^{t} I_{i}, X\right)=0$
c. $\operatorname{Ext}_{A}^{1}\left(\tau^{t} I_{i}, X\right)=0$.

We consider various possibilities, which dependend of the form of $t$, according to the list of the statement of 6.5 .

(1) Let $t \geq 1$ such that $t \equiv p-1(\bmod p)$ and $q \mid t$. Then, by 6.2 , we have

$$
\left(\operatorname{Supp} \tau^{-t} \mathcal{F}\right)^{\prime} \cap\left(\operatorname{Supp} \tau^{-t} \mathcal{G}\right)^{\prime}=\left(\operatorname{Supp} \tau^{-(p-1)} \mathcal{F}\right)^{\prime} \cap\left(\operatorname{Supp} \tau^{-t} \mathcal{G}\right)^{\prime}=\{p-1\} .
$$

We observe that $\operatorname{Hom}_{A}\left(\tau^{t} I_{p}, \tau^{t} I_{p-1}\right) \cong \operatorname{Hom}_{A}\left(I_{p}, I_{p-1}\right) \cong 0$, because $I_{p}$ has no $S_{p-1}$ as composition factor. Moreover, by $6.3(2), \operatorname{Ext}_{A}^{1}\left(\tau^{t} I_{p}, \tau^{t} I_{p-1}\right)=0$. Then $\left(\tau^{t} I_{p-1}, F, G, \tau^{t} I_{p}\right)$ is a s.s.

(2) Let $t \geq 1$ such that $t \equiv q-1(\bmod q)$ and $p \mid t$. By 6.2 we have that

$$
\left(\operatorname{Supp} \tau^{-t} \mathcal{F}\right)^{\prime} \cap\left(\operatorname{Supp} \tau^{-t} \mathcal{G}\right)^{\prime}=\left(\operatorname{Supp} \tau^{-t} \mathcal{F}\right)^{\prime} \cap\left(\operatorname{Supp} \tau^{-(q-1)} \mathcal{G}\right)^{\prime}=\{p+q-2\} .
$$

On the other hand, provided that $I_{1}$ has no $S_{p+q-2}$ as composition factor then $\operatorname{Hom}_{A}\left(\tau^{t} I_{1}, \tau^{t} I_{p+q-2}\right) \cong$ $\operatorname{Hom}_{A}\left(I_{1}, I_{p+q-2}\right) \cong 0$.

Finally by 6.3 it follows that $\operatorname{Ext}_{A}^{1}\left(\tau^{t} I_{1}, \tau^{t} I_{p+q-2}\right)=0$. Therefore $\left(\tau^{t} I_{p+q-2}, F, G, \tau^{t} I_{1}\right)$, with $t \geq 1$ such that $t \equiv q-1(\bmod q)$ and $p \mid t$, is a c.s.s..

(3) Let $t \geq 1$ such that $t \equiv p-1(\bmod p)$ eand $t \equiv q-1(\bmod q)$. By 6.2 we have that

$$
\left(\operatorname{Supp} \tau^{-t-1} \mathcal{F}\right)^{\prime} \cap\left(\operatorname{Supp} \tau^{-t-1} \mathcal{G}\right)^{\prime}=(\operatorname{Supp} \mathcal{F})^{\prime} \cap(\operatorname{Supp} \mathcal{G})^{\prime}=\{0, p+q-1\} .
$$

From the Auslander formula it may be concluded that

$$
\operatorname{Hom}_{A}\left(\tau^{t} I_{0}, \tau^{t+1} I_{p+q-1}\right) \cong \operatorname{Hom}_{A}\left(I_{0}, \tau I_{p+q-1}\right) \cong D \operatorname{Ext}_{A}^{1}\left(I_{p+q-1}, I_{0}\right)=0
$$

and that

$$
\operatorname{Ext}_{A}^{1}\left(\tau^{t} I_{0}, \tau^{t+1} I_{p+q-1}\right) \cong D \operatorname{Hom}_{A}\left(\tau^{t+1} I_{p+q-1}, \tau^{t+1} I_{0}\right) \cong \operatorname{Hom}_{A}\left(I_{p+q-1}, I_{0}\right)=0 .
$$

Consequently $\left(\tau^{t+1} I_{p+q-1}, F, G, \tau^{t} I_{0}\right)$, with $t \geq 1$ such that $t \equiv q-1(\bmod q)$ and $t \equiv p-1(\bmod p)$ is a s.s.

(4) Let $t \geq 1$ such that $t \equiv p-1(\bmod p)$ and $t \equiv q-1(\bmod q)$. We show that $\left(\tau^{t-p+1} I_{q-1}, F, G, \tau^{t} I_{p+q-1}\right)$ is a c.s.s. over $A$. By 6.2 follows that

$$
\left(\operatorname{Supp} \tau^{-t+p-1} \mathcal{F}\right)^{\prime} \cap\left(\operatorname{Supp} \tau^{-t+p-1} \mathcal{G}\right)^{\prime}=(\operatorname{Supp} \mathcal{F})^{\prime} \cap\left(\operatorname{Supp} \tau^{-(q-p)} \mathcal{G}\right)^{\prime}=\{q-1\} .
$$

Thus, $\operatorname{Hom}_{A}\left(\tau^{t} I_{p+q-1}, \tau^{t-(p-1)} I_{q-1}\right) \cong \operatorname{Hom}_{A}\left(\tau^{p-1} I_{p+q-1}, I_{q-1}\right)=0$, because by 6.10 (5) we have that $\left[\tau^{p-1} I_{p+q-1}: S_{q-1}\right]=0$. Moreover by $6.3(2)$, we have that $\operatorname{Ext}_{A}^{1}\left(\tau^{t} I_{p+q-1}, \tau^{t-(p-1)} I_{q-1}\right)=0$.

(5) Let $t \geq 1$ such that $t \equiv r(\bmod p), 0<r<p-1$ and $t \equiv q-1(\bmod q)$ and $X \cong \tau^{t-r} I_{p+q-r-2}$. First, by 6.2 , we have that

$$
\begin{aligned}
\left(\operatorname{Supp} \tau^{-(t-r)} \mathcal{F}\right)^{\prime} \cap\left(\operatorname{Supp} \tau^{-(t-r)} \mathcal{G}\right)^{\prime} & =(\operatorname{Supp} \mathcal{F})^{\prime} \cap\left(\operatorname{Supp} \tau^{-(t-r)} \mathcal{G}\right)^{\prime} \\
& =\left(\operatorname{Supp} \tau^{-(q-1-r)} \mathcal{G}\right)^{\prime} \\
& =\{p+q-r-2\}
\end{aligned}
$$


Furthermore, $\operatorname{Hom}_{A}\left(\tau^{t} I_{r+1}, \tau^{t-r} I_{p+q-r-2}\right) \cong \operatorname{Hom}_{A}\left(\tau^{r} I_{r+1}, I_{p+q-r-2}\right)=0$, because, by 6.10 $(5), \tau^{r} I_{r+1}$ has no $S_{p+q-r-2}$ as composition factor. Finally, by $6.3(2)$, we have that $\operatorname{Ext}_{A}^{1}\left(\tau^{t} I_{r+1}, \tau^{t-r} I_{p+q-r-2}\right)=0$. Then $\left(\tau^{t-r} I_{p+q-r-2}, F, G, \tau^{t} I_{r+1}\right)$ is a c.s.s. over $A$.

(6) Let $t \geq 1$ such that $t \equiv r(\bmod q), 0<r<q-1$ and $t \equiv p-1(\bmod p)$. To complete the s.s. $\left(F, G, \tau^{t} I_{p+r}\right)$ we consider two situations:

- Suppose that $r<p$. Let $X \cong \tau^{t-r} I_{p-(r+1)}$. We have that

$$
\begin{aligned}
\left(\operatorname{Supp} \tau^{-(t-r)} \mathcal{F}\right)^{\prime} \cap\left(\operatorname{Supp} \tau^{-(t-r)} \mathcal{G}\right)^{\prime} & =\left(\operatorname{Supp} \tau^{-(p-1-r)} \mathcal{F}\right)^{\prime} \cap(\operatorname{Supp} \mathcal{G})^{\prime} \\
& =\left(\operatorname{Supp} \tau^{(r+1)} \mathcal{F}\right)^{\prime} \\
& =\{p-(r+1)\} .
\end{aligned}
$$

Since $\left[\tau^{r} I_{p+r}: S_{p-(r+1)}\right]=0$, by $6.10(5)$, then

$$
\operatorname{Hom}_{A}\left(\tau^{t} I_{p+r}, \tau^{t-r} I_{p-(r+1)}\right) \cong \operatorname{Hom}_{A}\left(\tau^{r} I_{p+r}, I_{p-(r+1)}\right)=0 .
$$

By 6.3. (2), we have that $\operatorname{Ext}_{A}^{1}\left(\tau^{t} I_{p+r}, \tau^{t-r} I_{p-(r+1)}\right)=0$. Hence $\left(\tau^{t-r} I_{p-(r+1)}, F, G, \tau^{t} I_{p+r}\right)$ with $t \equiv r(\bmod q), 0<r<q-1, t \equiv p-1(\bmod p)$ and $r<p$ is a s.s..

- Suppose that $r \geq p$ and let $X \cong \tau^{t-(p-1)} I_{r}$. Then we have that

$$
\begin{aligned}
\left.\operatorname{Supp} \tau^{-[t-(p-1)]} \mathcal{F}\right)^{\prime} \cap\left(\operatorname{Supp} \tau^{-[t-(p-1)]} \mathcal{G}\right)^{\prime} & =\left(\operatorname{Supp} \tau^{-[r-(p-1)]} \mathcal{G}\right)^{\prime} \\
& =\{p+(r-p+1)-1\} \\
& =\{r\} .
\end{aligned}
$$

According to 6.10 (5), we have that

$$
\operatorname{Hom}_{A}\left(\tau^{t} I_{p+r}, \tau^{t-(p-1)} I_{r}\right) \cong \operatorname{Hom}_{A}\left(\tau^{p-1} I_{p+r}, I_{r}\right) \cong 0
$$

and by 6.3 we have that $\operatorname{Ext}_{A}^{1}\left(\tau^{t} I_{p+r}, \tau^{t-(p-1)} I_{r}\right)=0$. That is the sequence $\left(\tau^{t-(p-1)} I_{r}, F, G, \tau^{t} I_{p+r}\right)$ with $t \equiv r(\bmod q), 0<r<q-1, t \equiv p-1(\bmod p)$ and $r \geq p$ is a s.s.

Finally, 6.8 and 6.10 together are the main result of this section, which establish the complete list of c.s.s. of the form $(X, F, G, Y)$.

Theorem 6.11. Let $A=K \Delta$. The complete list of s.s. $(X, F, G, Y)$ is as follows:

(1) $\left(S_{p+q-1}, F, G, P_{0}\right)$.

(2) $\left(X, F, G, P_{p+q-1}\right)$, where

$$
X \cong\left\{\begin{array}{l}
\tau^{-p+1} P_{0}, \text { if } p=q \\
\tau^{-p+1} P_{q-1}, \text { if } p \neq q .
\end{array}\right.
$$

(3) $\left(X, F, G, \tau^{-t} P_{p+q-1}\right)$ with $t \geq 1$ such that $p \mid t$ and $q \mid t$, where

$$
X \cong\left\{\begin{array}{l}
\tau^{-t-p+1} P_{0}, \text { if } p=q \\
\tau^{-t-p+1} P_{q-1}, \text { if } p \neq q .
\end{array}\right.
$$

(4) $\left(\tau^{-t+1} P_{p+q-1}, F, G, \tau^{-t} P_{0}\right)$, with $t \geq 1$ such that $p \mid t$ e $q \mid t$.

(5) $\left(\tau^{-t-(p-r-1)} P_{q+r-1}, F, G, \tau^{-t} P_{p-r}\right)$, with $t \geq 1$ such that $q \mid t$ and $r$ such that $t \equiv r(\bmod p)$ and $1 \leq r \leq p-1$.

(6) $\left(X, F, G, \tau^{-t} P_{p+q-r-1}\right)$, with $t \geq 1$ such that $p \mid t$ and $r$ such that $t \equiv r(\bmod q)$ and $1 \leq r \leq q-1$.

$$
X \cong\left\{\begin{array}{l}
\tau^{-t-q+r+1} P_{p-q+r}, \text { if } p \leq q-r \\
\tau^{-t-p+1} P_{q-r-1}, \text { if } p>q-r .
\end{array}\right.
$$

(7) $\left(\tau^{t} I_{p-1}, F, G, \tau^{t} I_{p}\right)$, with $t \geq 1$ such that $t \equiv p-1(\bmod p)$ and $q \mid t$.

(8) $\left(\tau^{t} I_{p+q-2}, F, G, \tau^{t} I_{1}\right)$, with $t \geq 1$ such that $t \equiv q-1(\bmod q)$ and $p \mid t$.

(9) $\left(\tau^{t+1} I_{p+q-1}, F, G, \tau^{t} I_{0}\right)$, with $t \geq 1$ such that $t \equiv p-1(\bmod p)$ and $t \equiv q-1(\bmod q)$.

(10) $\left(\tau^{t-p+1} I_{q-1}, F, G, \tau^{t} I_{p+q-1}\right)$, with $t \geq 1$ such that $t \equiv p-1(\bmod p)$ and $t \equiv q-1(\bmod q)$.

(11) $\left(\tau^{t-r} I_{p+q-r-2}, F, G, \tau^{t} I_{r+1}\right)$, with $t \geq 1$ such that $t \equiv r(\bmod p), 0<r<p-1$ e $t \equiv q-1(\bmod q)$. 
(12) $\left(X, F, G, \tau^{t} I_{p+r}\right)$, with $t \geq 1$ such that $t \equiv r(\bmod q), 0<r<q-1$ and $t \equiv p-1(\bmod p)$, where

$$
X \cong\left\{\begin{array}{l}
\tau^{t-r} I_{p-(r+1)}, \text { if } r<p \\
\tau^{t-(p-1)} I_{r}, \text { if } r \geq p
\end{array}\right.
$$

\section{ACKNOWLEDGMENTS}

The first named author thanks CAPES and CNPq for financial support, during her PhD. The authors thank Maria Izabel Ramalho Martins for helpful discussions. The first named author also thanks her friend

Heily Wagner. The second author thanks CNPq for the research grant. Both authors used a tematic grant from Fapesp, to visit Marcelo Lanzilotta in Universidad de la República, Uruguay, they thank Fapesp for support and Marcelo for the warm hospitality.

\section{REFERENCES}

[1] Baer, D. (1989). A note on wild quiver algebras and tilting modules. Comm. in Algebra 17: 751-757.

[2] Bondal, A. I., Kapranov, M. M. (1989). Representable functors, Serre functors and reconstructions. Izv. Akad. Nauk SSSR Ser. Math. 53: 1183-1205, 1337(Russian). English translation in Math. USSR-Izv. 35 (1990), 519-541.

[3] Crawley-Boevey, W. Exceptional sequences of representations of quivers. In Representations of algebras, Proc. Ottawa 1992, eds V. Dlab and H. Lenzing, Canadian Math. Soc. Conf. Proc. 14 (Amer. Math. Soc., 1993), 117-124.

[4] Erdman, K., Sáenz, C. (2003). On standardly stratified algebras. Comm. in Algebra 31:3429-3446.

[5] Gorodentsev, A. L., Rudakov A. N. (1987). Exceptional vector bundles on projective spaces. Duke. Math. J. 54:115-130.

[6] Marcos, E., Mendoza O., Sáenz, C. (2004). Stratifying systems via relative simple modules. J. Algebra 280: $472-487$.

[7] Marcos, E., Mendoza O., Sáenz, C. (2005). Stratifying systems via relative projetive modules. Comm. in Algebra 33:15591573.

[8] Ringel, C. M. (1991). The category of good modules over a quasi-hereditary algebras has almos split sequences. Math. Z. 208:209-223.

[9] Rudakov, A. N. (1990). Exceptional collections, mutations and helices. In: Rudakov, A. N. et al, ed. Helices and vector bundles. London Math. Soc. Lecture Note Ser., 148. Cambridge Univ. Press, pp 1-6.

[10] Simson, D., Skowronski, A. (2007). Elements of Representation Theory of Associative Algebras. Vol 2: Tubes and Conceladed Algebras of Euclidean type. Series: London Math. Soc. Student Texts 71. Cambridge Univ. Press.

[11] Simson, D., Skowronski, A. (2007). Elements of Representation Theory of Associative Algebras. Vol 3: RepresentationInfinite Tilted Algebras. Series: London Math. Soc. Student Texts 72. Cambridge Univ. Press.

USP, Instituto de Matemática e Estatística

Rua de Matão 1010, Cidade Universitaria, São Paulo-SP

E-MAIL: PACA@IME.USP.BR

E-MAIL: ENMARCOS@IME.USP.BR 